\title{
CAVITY AND WAKE FLOWS
}

\author{
Th. YAO-TsU WU \\ California Institute of Technology, Pasadena, California
}

\section{Introduction}

The phenomenon of wake formation behind a body moving through a fluid, and the associated resistance of fluids, must have been one of the oldest experiences of man. From an analytical point of view, it is also one of the most difficult problems in fluid mechanics. Rayleigh, in his 1876 paper, observed that "there is no part of hydrodynamics more perplexing to the student than that which treats of the resistance of fluids." This insight of Rayleigh is so penetrating that the march of time has virtually left no mark on its validity even today, and likely still for some time to come.

The first major step concerning the resistance of fluids was made over a century ago when Kirchhoff (1869) introduced an idealized inviscid-flow model with free streamlines (or surfaces of discontinuity) and employed (for steady, plane flows) the ingenious conformal-mapping technique that had been invented a short time earlier by Helmholtz (1868) for treating twodimensional jets formed by free streamlines. This pioneering work offered an alternative to the classical paradox of D'Alembert (or the absence of resistance) and laid the foundation of the free-streamline theory. We appreciate the profound insight of these celebrated works even more when we consider that their basic idea about wakes and jets, based on a construction with surfaces of discontinuity, was formed decades before laminar and turbulent flows were distinguished by Reynolds (1883), and long before the fundamental concepts of boundary-layer theory and flow separation were established by Prandtl (1904a).

However, there have been some questions raised in the past, and still today, about the validity of the Kirchhoff flow for the approximate calculation of resistance. Historically there is little doubt that in constructing the flow model Kirchhoff was thinking of the wake in a single-phase fluid, and not at all of the vapor-gas cavity in a liquid; hence the arguments, both for and against the Kirchhoff flow, should be viewed in this light. On this basis, an important observation was made by Sir William Thomson, later Lord Kelvin (see Rayleigh 1876) "that motions involving a surface of separation are unstable" (we infer that instability here includes the viscous effect). Regarding this comment Rayleigh asked "whether the calculations of resistance are materially affected by this circumstance as the pressures experienced must be nearly independent of what happens at some distance in the rear of the obstacle, where the instability would first begin to manifest itself." 
This discussion undoubtedly widened the original scope, brought the wake analysis closer to reality, and hence should influence the course of further developments. An expanded discuision essentially along these lines was given by Levi-Civita (1907) and was included in the survey by Goldstein (1969).

Another point of fundamental importance is whether the Kirchhoff flow is the only correct Euler (or outer) limit of the Navier-Stokes solution to a steady flow at high Reynolds numbers. If so, then a second difficulty arises, a consequence of the following argument: We know that the width of the Kirchhoff wake grows parabolically with the downstream distance $x$, at a rate independent of the (kinematic) viscosity $\nu$. If Prandtl's boundary-layer theory is then applied to smooth out the discontinuity (i.e. the vortex sheet) between the wake and the potential flow, one obtains a laminar shear layer whose thickness grows like $(\nu x / U)^{1 / 2}$ in a free stream of velocity $U$. Hence, for sufficiently small $\nu / U$ the shear layers do not meet, so that the wake bubble remains infinitely long at a finite Reynolds number, a result not supported by experience. (For more details see Lagerstrom 1964, before p. 106, 131; Kaplun 1967, Part II.) The weaknesses in the above argument appear to lie in the two primary suppositions that, first, the free shear layer enveloping the wake would remain stable indefinitely, and second (perhaps a less serious one), the boundary-layer approximation would be valid along the infinitely long wake boundary. Reattachment of two turbulent shear layers, for instance, is possible since their thickness grows linearly with $x$.

By and large, various criticisms of the Kirchhoff flow model have led to constructive refinements of the free-streamline theory rather than to a weakening of the foundation of the thecry as a valuable idealization. The major development in this direction has been based on the observation that the wake bubble is finite in size at high Reynolds numbers. (The wake bubble, or the near-wake, means, in the ordinary physical sense, the region of closed streamlines behind the body as characterized by a constant or nearly constant pressure.) To facilitate the mathematical analysis of flows with a finite wake bubble, a number of potential-flow models have been introduced to give the near-wake a definite configuration as an approximation to the inviscid outer flow. These theoretical models will be discussed explicitly later. It suffices to note here that all these models, even though artificial to various degrees, are aimed at admitting the near-wake pressure coefficient as a single free parameter of the flow, thus providing a satisfactory solution to the state of motion in the near part of the wake attached to the body. On the whole, their utility is established by their capability of bringing the results of potential theory of inviscid flows into better agreement with experimental measurements in fluids of small viscosity.

The cavity flow also has a long, active history. Already in 1754, Euler, in connection with his study of turbines, realized that vapor cavitation may likely occur in a water stream at high speeds. In investigating the cause of the racing of a ship propeller, Reynolds (1873) observed the phenomenon of 
cavitation at the propeller blades. After the turn of this century, numerous investigations of cavitation and cavity flows were stimulated by studies of ship propellers, turbomachinery, hydrofoils, and other engineering developments. Important concepts in this subject began to appear about fifty years ago. In an extensive study of the cavitation of water turbines, Thoma (1926) introduced the cavitation number (the underpressure coefficient of the vapor phase) as the principal similarity parameter, which has ever since played a central role in small-bubble cavitation as well as in well-developed cavity flows. Applications of free-streamline theory to finite-cavity flows have attracted much mathematical interest and also provided valuable information for engineering purposes. Although the wake interpretation of the flow models used to be standard, experimental verifications generally indicate that the theoretical predictions by these finite-wake models are satisfactory to the same degree for both wake and cavity flows. This fact, however, has not been widely recognized and some confusion still exists. As a possible explanation, it is quite plausible that even for the wake in a single-phase flow, the kinetic energy of the viscous flow within the wake bubble is small, thus keeping the pressure almost unchanged throughout.

Although this review gives more emphasis to cavity flows, several basic aspects of cavity and wake flows can be effectively discussed together since they are found to have many important features in common, or in close analogy. This is in spite of relatively minor differences that arise from new physical effects, such as gravity, surface tension, thermodynamics of phase transition, density ratio and viscosity ratio of the two phases, etc., that are intrinsic only to cavity flows. Based on this approach, attempts will be made to give a brief survey of the physical background, a general discussion of the free-streamline theory, some comments on the problems and issues of current interest, and to point out some basic problems yet to be resolved. In view of the vast scope of this subject and the voluminous literature, efforts will not be aimed at completeness, but rather on selective interests. Extensive review of the literature up to the 1960 s may be found in recent expositions by Birkhoff \& Zarantonello (1957), Gilbarg (1960), Gurevich (1961), Wehausen (1965), Sedov (1966), Wu (1968), Robertson \& Wislicenus (1969), and Woods (1961).

\section{Physical Background}

Some main features of wake and cavity flows behind simple bodies such as the flat plate, wedges, circular cylinder, sphere, and cones have been observed experimentally in the various stages of their development over a wide range of the Reynolds number (see, e.g., Roshko 1954b, 1955, Lighthill 1963, Roshko \& Fiszdon, 1967). A brief description of the actual circumstances is given here in the hope that what we may learn from them can be of value in gaining a deeper understanding of the mechanism of wake and cavity flows.

The wake flow past a bluff body manifests itself at a sufficiently high Reynolds number. The dividing streamlines first separate from the body to form 
free shear layers, enveloping a relatively slow recirculating flow region-the near-wake. This region is characterized by a nearly constant wake underpressure $p_{c}$, which is less than the free stream pressure $p_{\infty}$, as a rule. To cite a specific case, we review the measurements by Arie \& Rouse (1956) of the velocity, pressure, and turbulence behind a normal plate $(3 \mathrm{in.} \mathrm{high)} \mathrm{in} \mathrm{a}$ wind tunnel with a stream of low turbulence intensity of 1.5 percent, and with a maximum velocity $U$ of $50 \mathrm{f} / \mathrm{s}$, corresponding to a plate Reynolds number of about $7.4 \times 10^{4}$. With a central splitter plate placed in the downstream, the wake bubble is about 17 times the plate height (above the splitter plate), and the underpressure coefficient is nearly constant $(\sigma \simeq 0.57)$ until the rear end of the near-wake is approached. A rapid transverse variation of the mean longitudinal velocity marks the location of the curved free shear layer on the bubble boundary. The free shear layer was found to become fully turbulent at a short distance from the point of separation, the maximum turbulence level reaching some 25 percent of the free-stream velocity at a station only four plate lengths from that point. At first, the longitudinal scale of turbulence appears to be considerably greater than the transverse owing to the strong shear, but this difference gradually decreases as the vorticity diffuses and the shear layer broadens further downstream. The strength of turbulent mixing is indicated by the large cross-correlation coefficient $\left(\overline{u^{\prime} v^{\prime}} /\left(\overline{u^{\prime 2} v^{\prime 2}}\right)^{1 / 2} \simeq-0.6\right.$, as deduced from the data (which is somewhat greater than that of the turbulent flow next to a wall); this strong mixing induces a recirculating flow within the wake bubble. As clearly indicated by these results, the original surface of discontinuity in the flow velocity is highly unstable, and the transfer of energy between the laminar and turbulent components takes place very rapidly. These two features, together with the fact that both the inertial force and turbulent friction are of equal importance in the free shear layer, render the problem difficult for mathematical analysis. The curvature effect of the free shear layer and the effect due to finite width of the near wake on the rate of growth of the shear layer seem to be still unknown. However, it is worth noting from the data of Arie and Rouse that the total width, $2 \delta$ say, of the curved, turbulent shear layer grows linearly with the streamwise distance $x$, at the rate of $d \delta / d x \simeq 0.1$, which is well in agreement with the case of straight free shear layer calculated by Görtler (1942), and measured by Reichardt (1942). The results of Arie and Rouse are consistent with those of Liepmann \& Laufer (1947) in their investigation of free turbulent mixing.

As the closure of the near-wake is approached, the fluctuations grow as the nonuniformity of the stresses within the two shear layers (from two sides of the body) increases. Consequently, these shear layers become unstable, rolling up in to large vortices. Roshko \& Fiszdon (1967) found experimentally that the reattachment point of the mean streamlines, which marks the closure of the near-wake, corresponds to the position in the unsteady flow where the vortices complete their growth and then break away. The shedding of these vortices (alternately on each side of a two-dimensional body) may appear at 
a definite frequency, creating a regular Kármán vortex sheet; at higher Reynolds numbers it may occur so violently as to generate directly an irregular, turbulent flow. The flow in such a region, being vortical and turbulent, is called the turbulent far-wake. The motion there is marked with an unsteadiness, and is stationary only in the gross mean. Experimental investigations of the periodic vortex shedding from a two-dimensional body (mainly for circular cylinders) have revealed a definite but rather weak dependence of the Strouhal number on the Reynolds number (Kovasznay 1949, Roshko 1954b, Goldburg et al 1965, Gerrard 1966, Gaster 1971). In contrast, there is little published work on incompressible wake structure behind threedimensional bodies; the few results available are mostly for disks and spheres. While the regular shedding of vortices from two-dimensional configurations can dominate the wake, its effect in simple axisymmetric arrangements is usually rather weak. However, when asymmetry is present as with an inclined disk (Calvert 1967b), very strong vortex shedding occurs. The vortices appear in the form of a chain of irregular rings, consecutive vortices being interlinked. They have little in common with the two-dimensional vortex street. The near-wake, on the other hand, is again of the recirculating bubble-flow type (Calvert 1967a).

Towards the end of the turbulent far-wake, the scale of turbulence diminishes owing to mixing and dissipation, reducing the flow eventually to an asymptotic laminar far-wake, where the thermal process finally takes over. Along the entire far-wake, the mean pressure increases gradually from the wake underpressure $p_{c}$ and ultimately recovers the main stream pressure $p_{\infty}$ far downstream.

The essential interaction between the body and the separated flow occurs in the region enclosing the body and its near-wake. However, the near-wake flow (its base pressure and flow pattern in particular) is in turn related to the state of motion in the far-wake. The overall flow pattern depends primarily on the Reynolds number, which prescribes the criterion for instability of the flow and hence provides an indication of the onset and location of transition from laminar to turbulent flow. The expository experimental study by Roshko \& Fiszdon (1967) for the circular cylinder illustrates vividly these salient features, which we summarize below. When the Reynolds number $R$ (based on diameter) lies between 1 and 50, the entire flow, including the wake bubble and the ensuing wake, is laminar and steady. In the region $50<R$ $<200$, the near-wake becomes unstable, oscillating periodically, though still laminar. First appearance of turbulence occurs near the closure point; the base pressure coefficient $C_{p_{b}}$, which is always negative, further decreases $\left(C_{p_{b}}\right.$ refers to the base point diametrically opposite the front stagnation point); a vortex street forms in the far-wake. For $200<R<1500$, turbulence near the closure point develops and spreads; periodic motion extends further downstream; $C_{p_{b}}$ becomes somewhat less negative. In the region $1500<R$ $<2 \times 10^{5}$, the laminar-to-turbulence transition point gradually moves upstream along the free shear layer; the separation point starts to move for- 
ward and the near-wake grows in size; $C_{p_{b}}$ decreases to a low value of about -1.0 ; the far-wake becomes irregular. At $R=R_{c}\left(\sim 5 \times 10^{5}\right)$, transition coincides with separation, begins to precede it, and therefore to postpone it. The flow undergoes first a laminar separation, followed by a reattachment to the cylinder, and then a turbulent separation to form a narrower near-wake. Then $C_{p_{b}}$ increases sharply to about -0.2 , resulting in a large fall in $C_{D}$, a phenomenon known as the drag crisis. In the higher range of $R\left(4 \times 10^{6}<R\right.$ $\left.<8 \times 10^{6}\right)$, transition further precedes the turbulent separation, and $C_{p_{b}}$ levels down to about -0.8 . The upper limit of the experiments to date is $R=8 \times 10^{6}$ (Roshko 1961), and beyond that the trend is not clear, particularly as $R \rightarrow \infty$. Batchelor (1956) argues that the length of the wake bubble would tend to a constant and $C_{D}$ would tend to zero as $R \rightarrow \infty$; but Imai (1957) and others contend that the bubble length would increase linearly with $R$, while the flow would asymptotically approach the Kirchhoff flow in this limit.

In addition to its own intrinsic instability, the level of unsteadiness of wake flow involves other complexities. One of them is the free-stream turbulence level; the surface condition (roughness and compliance of body surface) is another. Both may affect the location of transition-e.g., an increase of either of them tends to reduce $R_{c}$. Another important effect arises from the basic difference between the separation from a sharp corner (the fixed separation) and the separation at a point of a continuously curved body (the free separation). In general, the wake flows with fixed separation are steadier and the base pressure tends to be more uniform, whereas those past wellrounded bodies tend to have stronger oscillations. This is because the flow towards a sharp edge is always accelerated by a favorable pressure gradient, a state that is little affected by changes in Reynolds number. Still another factor is the entrainment mechanism., which can be influenced not only by the location of transition but also by additional constraints, such as a splitterplate inserted along the wake central. line. Such an extraneous device has a marked effect in suppressing the alternate shedding of vortices, and raises up the base pressure to such an exterit that the drag coefficient of a circular cylinder or a flat plate is reduced by about one-third of its value when the splitter plate is absent (Roshko 1954b, Arie \& Rouse 1956).

Although wake flows are practically always unsteady, their mean motion, obtained by averaging over times large compared to the characteristic period, appears steady. (Figure 1 is a sketch of an incompressible wake flow.) It is this mean motion that has bcen of wide interest and will constitute the principal part of our discussion. We note that in a nondimensional form, incompressible wake flows are characterized by the wake underpressure coefficient (or wake number)

$$
\sigma=\frac{p_{\infty}-p_{c}}{\rho U^{2} / 2}
$$

where $p_{\infty}$ is the pressure and $U$ the velocity of the free stream, $\rho$ the fluid density, and $p_{c}$ the mean base pressure in the near-wake. 


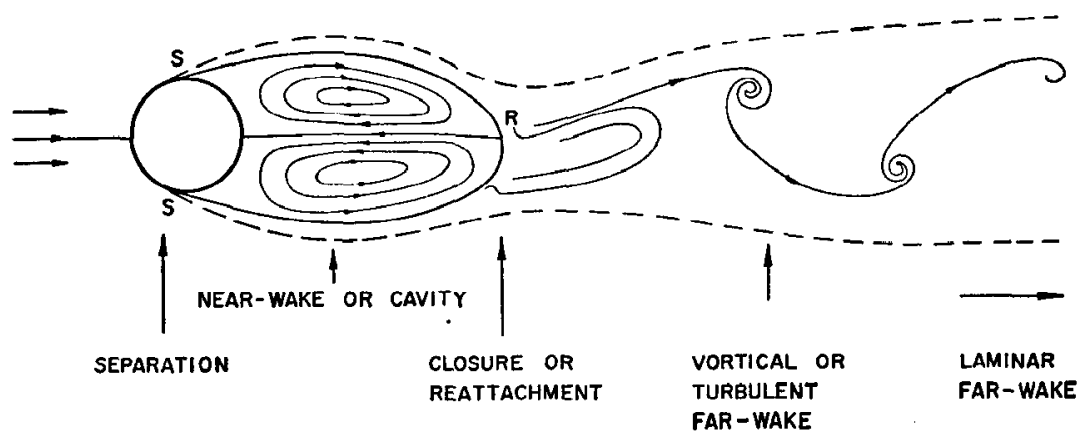

FiguRE 1. A streamline pattern in a developed wake or cavity flow. The streamlines inside the wake bubble or cavity represent their mean position.

When a bluff body moves in a liquid medium, such as water, cavitating flow may also have different flow patterns. In its initial stage, cavitation refers to the formation in a liquid flow of small bubbles, filled with vapor and gas, because of reduction in the local pressure. By increasing the flow speed and decreasing the free-stream pressure, local cavitation begins in a limited region where the minimum pressure reaches a value close to or less than the vapor pressure. If $p_{c}$ in Equation (1) is taken to be the mean pressure of the vapor-gas mixture in bubbles, averaged over a large number of bubbles, then the various stages of cavitating flows can also be characterized by the parameter $\sigma$, now called the cavitation number. The rate of cavitation in this inception stage depends on $\sigma$ as well as on various other physical factors, such as the concentration of nuclei (generally regarded as microscopic solid particles immersed in liquid), amount of dissolved gas, surface tension, various diffusion coefficients, and other thermodynamic effects. The mechanics of limited cavitation is beyond the present scope; reference is made to recent surveys by Plesset (1964), Eisenberg \& Tulin (1961), Robertson \& Wislicenus (1969), and Knapp et al (1970).

As $\sigma$ decreases, bubbles increase both in number and in size and coalesce to form larger bubbles. Eventually, for $\sigma$ sufficiently low (usually of order unity or less), a single, large cavity is formed in the wake of the body, enveloping a vapor-gas mixture of constant pressure, which now plays the role of $p_{c}$ in Equation (1).

Cavity flows and wake flows have many salient features in common or in close analogy. The cavity itself may be likened to the bubble shape of a near-wake. The motion of the gas-vapor mixture inside the cavity is also of the recirculating type, though its dynamic effect is much weaker because of the large differences in the density and viscosity of the two phases. At the closure of a two-dimensional cavity, the liquid flow resembles strong vortices, fluctuating and reattaching in the mean, often forming a liquid jet going upstream for some distance into the cavity; hence the name reentrant jet. This closure flow is similar to the vortical flow near the end of a wake bubble. 
Downstream of a cavity, the regular pattern of Kármán's vortex street has also been observed in an appropriate range of Reynolds number (Young \& Holl 1966); irregular turbulent mixing generally takes place at higher Reynolds numbers. Thus the far-wakes in these two cases are essentially the same. Furthermore, cavity flows, too, have some intrinsic unsteadiness, which depends in the same qualitative way on the physical factors we have already described for wake flows. The intensity of fluctuations can be especially violent in the "partial cavitation" of hydrofoils, that is, when the length of the cavity formed on the suction side of the lifting foil approaches the length of the foil. In this case, the closure vortices oscillate in the proximity of the trailing edge, apparently exciting certain modes of periodic disturbance. Perhaps the action of water impact of the reentrant jet on the body is also involved.

Experimental studies of cavity flows have been largely limited to measurements of the hydrodynamic forces and moments, and, to a lesser extent, of pressure distributions and some other details of the flow field. There is little published work on fundamental investigations of hydrodynamic stability, transition, separation, turbulence scales, relationship between the cavitation number and Reynolds number, and other physical effects. This is understandable on account of the evident difficulties with such watertunnel experiments, upon which I shall not elaborate. Part of such urgently needed information has come to light through a series of basic studies by Brennen $(1969 \mathrm{a}, \mathrm{b} ; 1970 \mathrm{a}, \mathrm{b})$ on the nature of the interfacial shear layer along the cavity boundary after its viscous separation from a number of axisymmetric headforms. For a spherical cap, the cavity surface for a short stretch, immediately after separation, invariably appeared smooth and glossy (Figure 2), suggesting a laminar separation even at the highest Reynolds number $\left(9 \times 10^{6}\right)$ attained. At a short distance away from separation, a system of surface waves appeared, growing in amplitude as they propagated downstream, until transition took place (at high speeds only) to make them turbulent. At lower tunnel speeds, these waves persisted along the length of cavity. Brennen (1970a) ascribed these waves to the growth of an instability in the separated laminar shear layer, the observed frequency being close to that predicted by inviscid Orr-Sommerfeld theory. Among the various headforms tested by Brennen, only the circular disk produced cavities completely clear and glossy at all speeds (unless the separation was forcibly made turbulent (Figure 2c,d)). The surface-tension effect, generally speaking, is at most secondary, as was shown (Brennen 1970b) by some results with a surfacetension reducing agent. Brennen (1969a) also investigated the dynamic balances of dissolved air and heat in natural cavity flows, describing how the air diffused into the cavity leaves it by entrainment near cavity closure.

Cavity separation becomes even more complex a phenomenon when it occurs at a slender or thin body, as compared to bluff bodies. Viscous separation of a cavity from a thin body may be preceded by a region of low pressure slightly less than $P_{q}$ in the cavity, so that the nuclei in the liquid 
A Annual Reviews

wWw.annualreviews.org/aronline

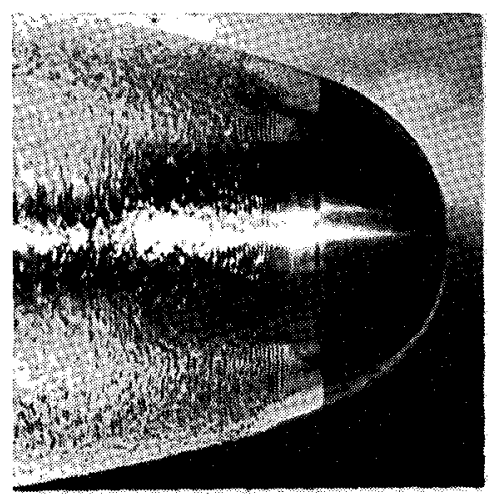

(a)

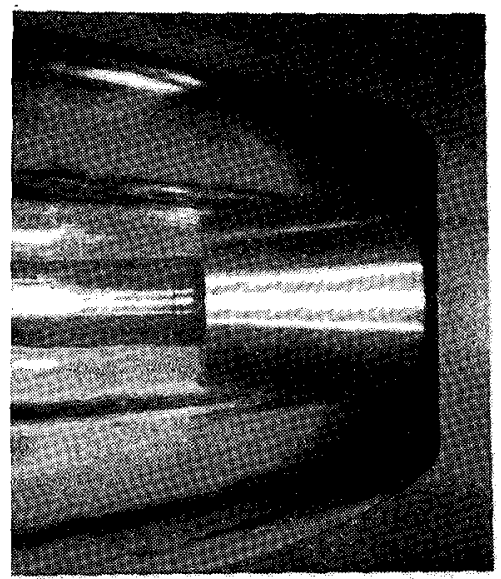

(c)

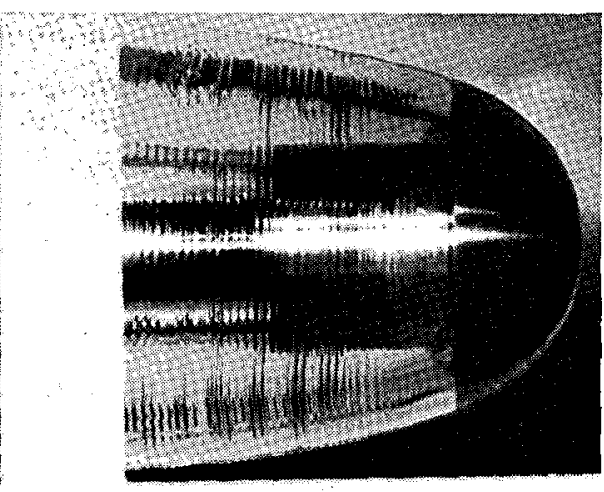

(b)

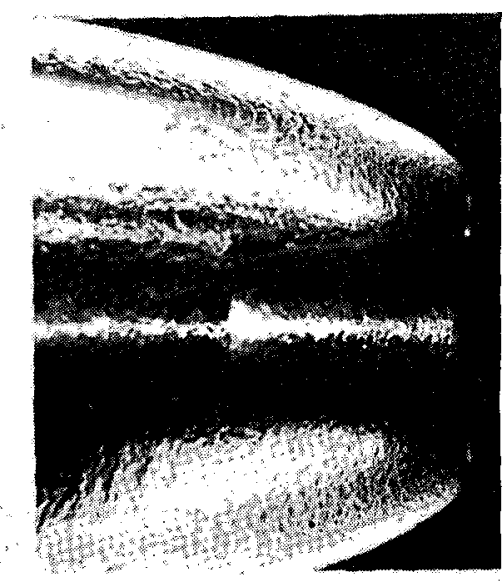

(d)

FIGURE 2. Flow patterns on the surface of gas-vapor cavities in water. (a) spherical cap, $U=20 \mathrm{ft} / \mathrm{sec}$; (b) spherical cap, $U=12 \mathrm{ft} / \mathrm{sec}$; (c) disk, $U=35 \mathrm{ft} / \mathrm{sec}$; (d) disk with ring on face, $U=30 \mathrm{ft} / \mathrm{sec}$ (Brennen 1970a). 
stream, if given enough time in transit, may induce early cavitation, giving rise to the so-called "nucleate separation" as opposed to the "smooth viscous cavity separation." This phenomenon has been investigated by Arakeri (1971) with cavitating hydrofoils.

All the above rudimentary description and comment merely serve to illustrate the complexities of the problem. In spite of many uncertainties still existing, the outstanding properties of wake and cavity flows are nevertheless useful for providing a sound physical basis for constructing theoretical models.

\section{Inviscid Outer Flow, the Free-Streamline Theory}

The inviscid approximation for cavity and wake flows at high Reynolds numbers is important for several reasons. First, this approximate theory is effective in providing satisfactory predictions of the hydrodynamic forces and moments for engineering purposes. From the point of view of assisting further basic developments, this Euler solution of the outer inviscid ffow is also needed for calculating the viscous flow in the near-wake, or cavity, and its neighborhood.

Some drastic simplifying assumptions are necessary to construct a theoretical model, whose validity, of course, can be justified only with experimental verifications. For cavity flows, it is usually assumed that the cavity and its ensuing wake are separated from the outer flow by free streamlines or surfaces, and that the outer flow is inviscid and irrotational. The same approximation applied to real wakes amounts to supposing that the shear layer around the near-wake is thin, that rolling up and mixing of trailing vortices are neglected, and that the mean steady flow is of primary interest. For completencss, we state the theory including the unsteady flow that may result from body motion and disturbances (of body scale) in the basic flow. The fluid is assumed for the present to be incompressible.

Under these assumptions, the flow has a velocity potential $\phi(x, t)$, satisfying the Laplace equation

$$
\nabla^{2} \phi=0
$$

The classical boundary conditions are as follows.

(i) On a solid surface, with its motion prescribed by $S(\mathbf{x}, t)=0$,

$$
\frac{d S}{d l}=S_{t}+(\nabla \phi) \cdot \nabla S=0 \quad \text { on } S(\mathbf{x}, t)=0
$$

(ii) On a free boundary, $F(\mathbf{x}, t)=0$ say, there are two conditions. The kinematic condition requires

$$
\frac{d F}{d t}=F_{t}+(\nabla \phi) \cdot \nabla F=0 \quad \text { on } F(\mathbf{x}, t)=0
$$


For the dynamic condition, the classical assumption (neglecting the effects of gravity and surface tension) is that the pressure $p$ is constant, say $p_{c}$, on the cavity (or near-wake) boundary. Hence, by the Bernoulli equation,

$$
\phi_{t}+\frac{1}{2}(\nabla \phi)^{2}=\frac{1}{2} U^{2}(1+\sigma) \quad \text { on } F(\mathrm{x}, t)=0
$$

$U$ being the free stream velocity and $\sigma$ the cavitation number defined by Equation (1). The cavity pressure $p_{c}$ is assumed to be the minimum pressure of the entire flow.

(iii) A point at which the free streamline of an inviscid flow leaves the body will be called a detachment point (either fixed or free); it is quite possible that the free detachment point of an inviscid flow is different from the free separation point of the corresponding viscous flow past the same smoothly curved body. Since the location of a free detachment point is not known a priori, a condition is required for its determination. The classical condition (originally introduced for plane flows) is the one due to Brillouin (1911) and Villat (1914), requiring that the curvature of a free streamline at free detachment be finite (the Villat-Brillouin condition). When this condition is satisfied, the streamline curvature is automatically equal to that of the body surface at the free detachment point (for this reason, free detachment is also called "smooth detachment" in the literature). The same condition can be extended to three-dimensional wakes and cavities. Necessity of this condition arises partly from the fact that the streamline curvature at a fixed detachment is generally infinite. This condition may thus seem to have a physically natural basis in the framework of potential theory. It may also be argued, however, that cavity separation, as well as the boundary-layer separation in wakes, is a viscous phenomenon. This is amply illustrated by the differences in the observed position of separation and the location of free detachment predicted by the potential theory for rounded bodies. More discussion of this point will be given later.

For finite cavities and wakes, it is further necessary to introduce a specific flow model so that the far-wake can assume a definite shape in this inviscid approximation. A number of theoretical models have been proposed and invariably employed first in some simple configurations (such as a normal flat plate or symmetric wedges) in plane flow. We describe below some of the most commonly used models with direct extension to curved bodies. Generalization to the three-dimensional case is immediate.

1. The Riabouchinsky image model. This was proposed by Riabouchinsky (1920). An "image" body is introduced at a certain distance downstream of the real body to make the cavity closed. Figure 3 a shows the choice of the image body being symmetric with respect to the centroid of the cavity. This is the simplest body-cavity-image system that, in the absence of circulation, will experience no net hydrodynamic force; other image shapes will either lead to nonexistence of the postulated potential flow or will require an appropriate circulation to make the solution possible. 


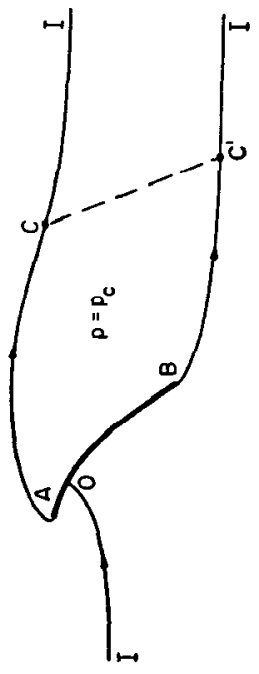

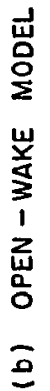

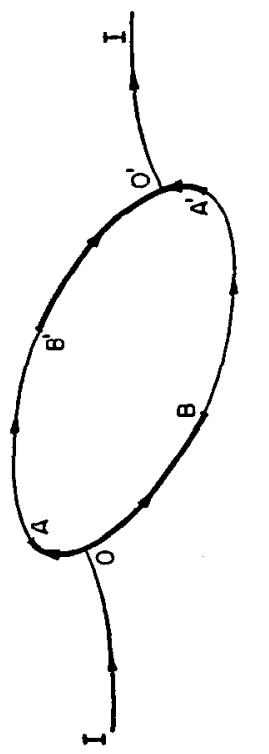

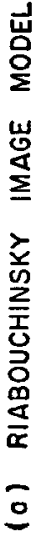
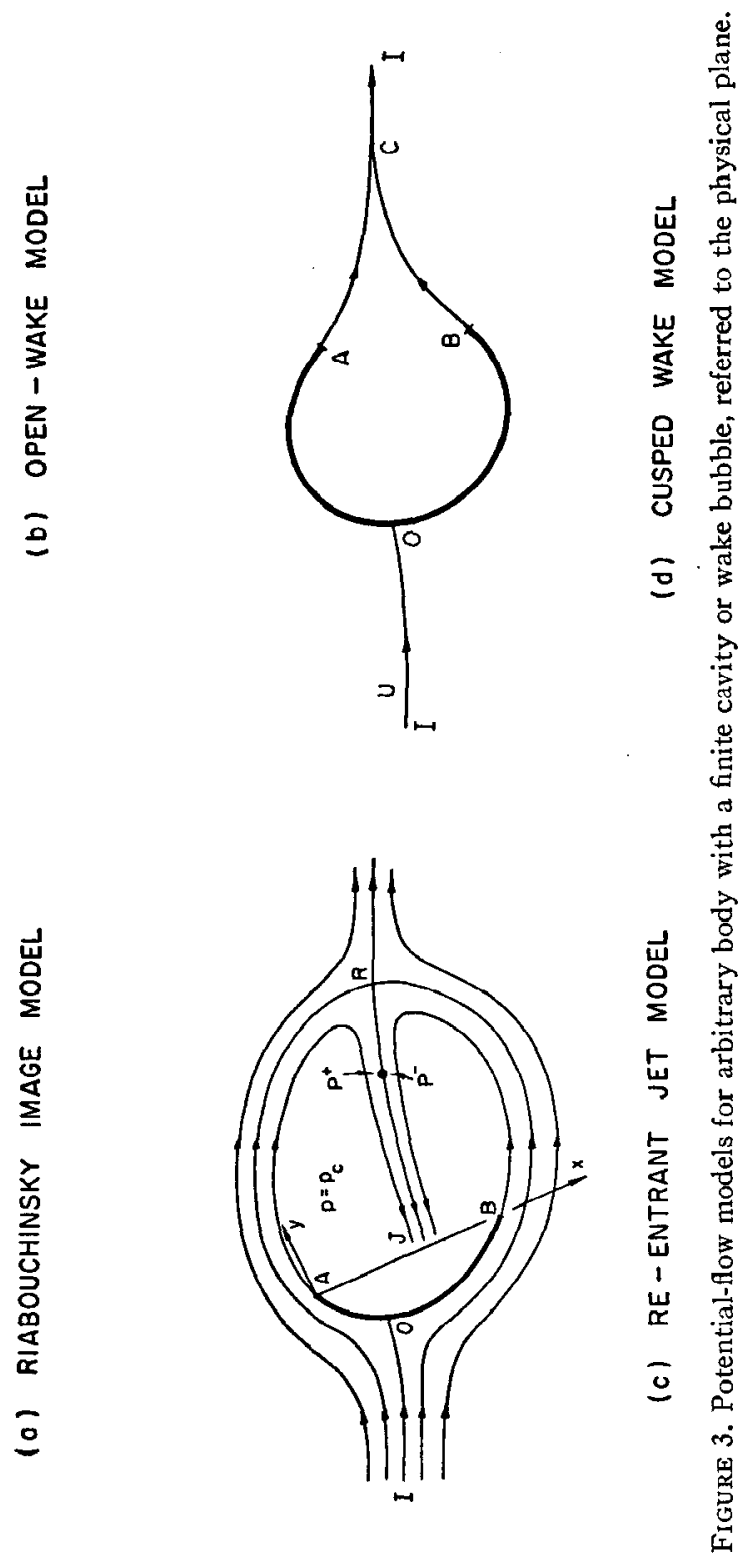
2. Open-wake model. This model was first introduced by Joukowsky (1890), in a fundamental work which apparently remained unnoticed for a long time. It was again proposed independently by McNown \& Yih (1953), Roshko (1954a), and Eppler (1954) for symmetric bodies in nonlifting flows. $\mathrm{Wu}$ (1962) modified the model in order to extend it to lifting flows past arbitrary bodies (Figure 3 ) by requiring that the undetermined velocities on the streamlines bounding the semi-infinite strip, which represents the far wake, are equal at equal values of the velocity potential. This strip thus models the actual turbulent mixing and viscous dissipation in a far-wake of constant momentum thickness.

3. The reentrant-jet model. This model seems to have been first suggested by Prandtl and $\mathrm{H}$. Wagner, and later formulated by Kreisel (1946) and independently by Efros (1946). In this model, the two dividing streamlines reverse direction, carrying a finite part of main stream into a jet which flows through the cavity (in an undetermined direction for lifting flows) and is continued mathematically into a second Riemann sheet.

4. Cusped-wake model. Brillouin (1911) appears to have been the first to discuss the finite cavity which is terminated with a cusp and especially the fact that the minimum pressure no longer falls on the cavity boundary. This model was applied to the circular cylinder by Southwell \& Vaisey (1946) and was taken as a realistic model for truncated airfoils by Lighthill (1945).

5. The Batchelor wake model. With wakes at high Reynolds numbers in mind, Batchelor (1956) proposed a closed wake model which encloses a pair of stationary viscous eddies of constant vorticity. Of particular concern was the uniform validity of possible wake models as the viscosity $\mu \rightarrow 0$. An outstanding property of this model is that the resistance of the fluid on the body vanishes in this limit.

6. Vortex models. Systems of stationary vortices are observed in the numerical calculations of wakes at low Reynolds number; for example, the early computations of Thom (1933) and Apelt (1957) show a pair of stationary vortices behind a circular cylinder up to a Reynolds number of 40. For high Reynolds numbers, various kinds of twin vortices, such as the single-spiral and double-spiral vortices, have been proposed as possible wake and cavity models by Weinig (1956) and Tulin (1964).

7. The Lavrentiev wake model. Lavrentiev (1962) proposed a closed wake-bubble model which terminates with a stagnation point at the closure and has a recirculating flow that encloses two separate regions of constant pressure. Its potential utility in the determination of viscous near-wake remains to be realized,

Aside from these models, still others have been tried out. For instance, Hopkinson (1898) considered the flow bounded by two plates and two free streamlines with interior sources and vortices. Artificial plates have been used to intercept free streamlines and to control the open wake width (Gadd 1962). The one-sided reentrant jet at the edge of a wedge or plate 
was treated by Cox \& Clayden (1958), Pykhteev (1960), and Cumberbatch (1961). These potential flow models all involve some sort of artifice in representing flows with a finite cavity or near-wake, thereby admitting the cavitation number $\sigma$ as a single free parameter. The need of an artifice, one kind or another, can be realized by the argument (Wu 1956) that in the framework of potential theory, the work done by the resistance of fluid on a moving body cannot be removed without at the same time removing momentum, or mass, or both. For this reason, the potential-flow models should not be expected to provide a good approximation of the far-wake as well as the near-wake, since the viscous dissipation in real fluids is a rather slow process.

Once the model is accepted, the mathematical problem becomes definite. These potential-flow problems, first of all, are nonlinear (due to the nonlinear conditions on the free boundary, whose location is unknown), though a linearized theory has been developed for thin bodies and hydrofoils (see Section 10). Another difficulty arises from the gravity effect in (two-phase) cavity flows, in particular when an unbounded water surface is present, as in planing-surface problems. For unsteady cavity flows generated by a timevarying free-stream and/or body motion, the problem is further complicated by the explicit dependence of the flow on its time history, as is clearly indicated by the free-boundary conditions [Equations (4) and (5)]. Because of these difficulties, only the special class of steady, plane flows (in the absence of gravity in case of cavity flows) has received an impressive development, since the powerful hodograph method and complex function theory are then at our disposal.

\section{Steady Plane Flows}

Mathematical analysis: the functional equations.-For a steady, incompressible, potential flow in the physical plane $z=x+i y$, the complex velocity potential $f=\phi+i \psi$ and the complex (conjugate) velocity $w=d f / d z=u-i v$ are analytic functions of $z$, and hence of each other. It is of ten more convenient to use, instead of $w$, the logarithmic hodograph variable

$$
\omega=\log \frac{q_{c}}{w}=\tau+i \theta, \quad \tau=\log \frac{q_{c}}{|w|}, \quad \theta=\tan ^{-1} \frac{v}{u}
$$

$q_{c}$ being the constant flow speed on the cavity boundary.

The analytical solution of a cavity or wake flow past an arbitrary body in classical free-streamline theory is most effectively determined in a parametric form: $f=f(\zeta), \omega=\omega(\zeta)$, where $\zeta=\xi+i \eta$ is a suitable complex variable. For a unified discussion of different flow models, the flow region is mapped conformally in to the lower half of the $\zeta$ plane, with the wetted body surface mapped into $\eta=0,-1<\xi<1$, the stagnation point into the origin $\zeta=0$, and the cavity or near-wake boundary into $\eta=0,|\xi|>1$. Since the flow region in the $f$ plane is always in the form of strips ( $\psi=$ const on both fixed and free boundaries), and may involve Riemann surfaces (de- 
pending on the flow model chosen, see Figure 4), the mapping $f=f(\zeta)$ can readily be effected by applying the generalized Schwarz-Christoffel transformation due to Gilbarg (1949). The results are given for four sample flow models, as follows:

$$
\begin{array}{rlrl}
\frac{d f}{d \zeta} & =A \zeta\left(\zeta-\zeta_{0}\right)^{-3 / 2}\left(\zeta-\bar{\zeta}_{0}\right)^{-3 / 2} & & \text { Riabouchinsky model } \\
& =A \zeta\left(\zeta-\zeta_{0}\right)\left(\zeta-\zeta_{0}\right)^{-2}\left(\zeta-\bar{\zeta}_{0}\right)^{-2} & & \text { open-wake model and } \\
& \text { cusped-wake model } \\
& =\frac{A \zeta\left(\zeta-\zeta_{R}\right)\left(\zeta-\bar{\zeta}_{R}\right)}{\left(\zeta-\zeta_{0}\right)^{2}\left(\zeta-\bar{\zeta}_{0}\right)^{2}\left(\zeta-\zeta_{J}\right)} & & \text { reentrant-jet model }
\end{array}
$$

where $A$ is a real constant, $\zeta_{0}$ corresponds to $f=\infty, \zeta_{c}$ to the constant pressure terminal $C$, and $\zeta_{R}$ and $\zeta_{J}$, to the rear stagnation point $R$ and the jet terminal $J$, respectively (see Figure 4 ); $\bar{\zeta}_{0}$ stands for the complex conjugate of $\zeta_{0}$.

Let the wetted solid boundary be given parametrically by

$$
x=x(s), \quad y=y(s) \quad(0<s<S)
$$

$s$ being the arc length along the body surface. with $s=0$ at point $A, s=S$ at point $B$. The boundary conditions for $\omega(\zeta)=\tau(\xi, \eta)+i \theta(\xi, \eta)$ then read

$$
\begin{array}{rlrl}
\theta^{-}(\xi) & \equiv \theta\left(\xi, 0^{-}\right)=\pi H(-\xi)+\hat{\beta}(s(\xi)) & & (-1<\xi<1) \\
\tau^{-}(\xi) \equiv \tau\left(\xi, 0^{-}\right)=0 & & (|\xi|>1)
\end{array}
$$

where $H(-\xi)=1$ for $\xi<0, H(-\xi)=0$ for $\xi>0$, and $\hat{\beta}(s)=\tan ^{-1}\left[y^{\prime}(s) / x^{\prime}(s)\right]$, the prime denoting ordinary differentiation. The solution of this RiemannHilbert problem is

$$
\begin{aligned}
\omega(\zeta) & =\omega_{0}(\zeta)+\omega_{1}(\zeta)+\omega_{c}(\zeta) \\
\omega_{0}(\zeta) & =\log \frac{1+i\left(\zeta^{2}-1\right)^{1 / 2}}{\zeta} \\
\omega_{1}(\zeta) & =\frac{1}{\pi i} \int_{-1}^{1}\left(\frac{\zeta^{2}-1}{1-t^{2}}\right)^{1 / 2} \frac{\beta(t) d t}{t-\zeta}
\end{aligned}
$$

where $\beta(\xi)=\hat{\beta}(s(\xi))$, the function $\left(\zeta^{2}-1\right)^{1 / 2}$ is analytic in the $\zeta$ plane cut from $\zeta=-1$ to 1 along $\eta=0$ so that it tends to $\zeta$ as $|\zeta| \rightarrow \infty$, and $\omega_{c}(\xi)$ stands for the complementary solution $\theta_{c}^{-}(\xi)=0$ for $|\xi|<1$ and $\tau_{c}-(\xi)=0$ for $|\xi|$ $>1$ ), which may possess interior singularities depending on the specific flow model used. In fact, we have $\omega_{c}(\zeta)=0$ for the Riabouchinsky, openwake, and cusped-wake models; and

$$
\omega_{c}(\zeta)=\log \frac{\left(t-\bar{t}_{R}\right)\left(t t_{R}-1\right)}{\left(t-t_{R}\right)\left(t \bar{t}_{R}-1\right)} \quad \zeta=\frac{1}{2}\left(t+\frac{1}{t}\right)
$$

for the reentrant jet model. 

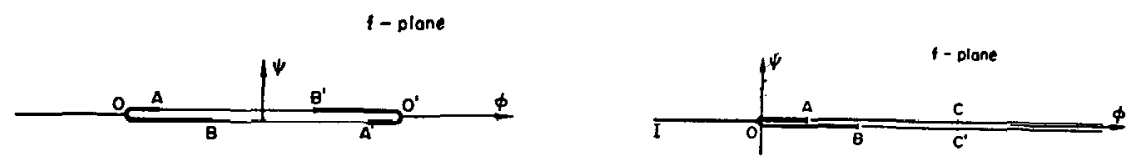

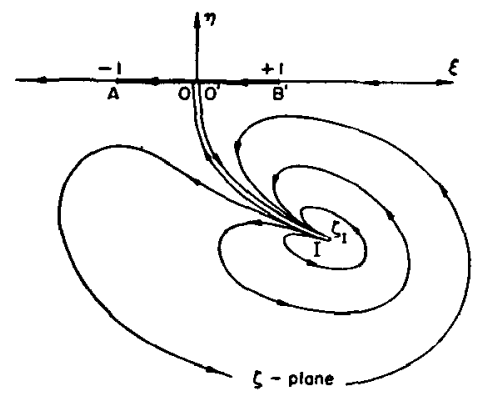

(0) RIABOUCHINSKY IMAGE MODEL
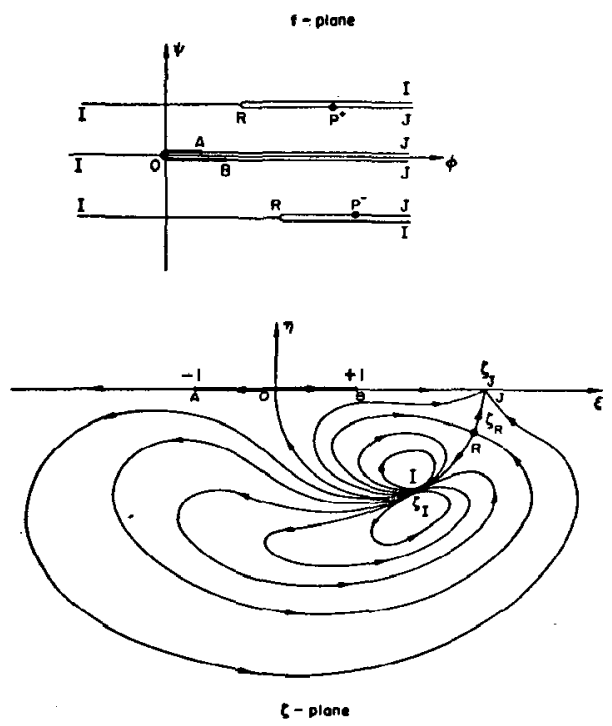

(c) RE-ENTRANT JET MODEL

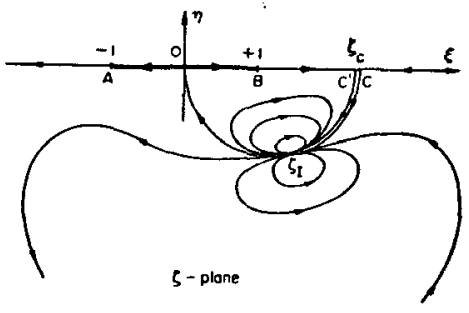

(b) OPEN - WAKE MODEL
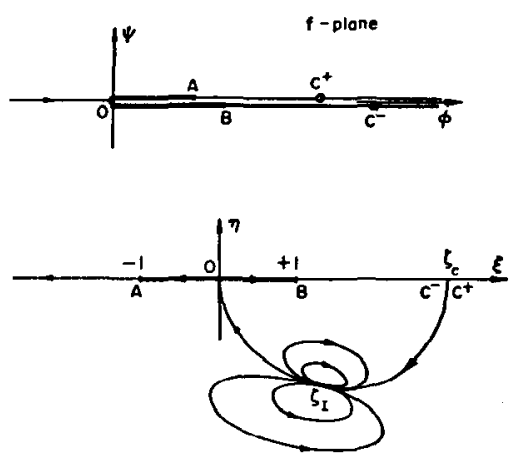

$\zeta$ - plane

FIGURE 4. Streamlines in the complex potential $f$ plane and a parametric $\zeta$ plane, the flow boundaries and designated points corresponding to those indicated in the respective flow models of Figure 3. 
The physical plane is now given by the quadrature

$$
z(\zeta)=\int \frac{1}{w(\zeta)} \frac{d f}{d \zeta} d \zeta
$$

The arc length $s(\xi)$, measured along the body surface from $\xi=-1$, is

$$
\begin{aligned}
& s(\xi)=\frac{A}{q_{c}} \int_{-1}^{\xi} e^{\Gamma(\xi)} \nu(\xi) d \xi \quad(-1<\xi<1) \\
& \Gamma(\xi)=-\frac{1}{\pi} \oint_{-1}^{1}\left(\frac{1-\xi^{2}}{1-t^{2}}\right)^{1 / 2} \frac{\beta(t) d t}{t-\xi} \\
& \nu(\xi)=\frac{1}{A} \exp \left[\omega_{0}^{-}(\xi)+\omega_{c}^{-}(\xi)\right] \frac{d f}{d \xi}
\end{aligned}
$$

The integral in Equation (16) assumes its Cauchy principal value. The total wetted arc length is specified as

$$
S=s(1)
$$

Moreover, the velocity condition at $z=\infty$, or $\zeta=\zeta_{0}$, requires that

$$
\omega\left(\zeta_{0}\right)=\log \frac{q_{c}}{U}+i \alpha=\frac{1}{2} \log (1+\sigma)+i \alpha
$$

where $\alpha$ is the incidence angle between the free stream and chord $A B$. This completes the formal solution of the Riabouchinsky flow. For the other models, however, further determining equations are required for the parameters $\zeta_{c}, \zeta_{R}$, and $\zeta_{J}$. They can be stated as follows: In the open-wake model, $f(\zeta)$ must be simply covered in the neighborhood of $\zeta=\zeta_{0}$. In both the cuspedwake and reentrant-jet flows, $z(\zeta)$ is required to be simply covered near $\zeta=\zeta_{0}$. Furthermore, there is left in the reentrant-jet flow the freedom of choice in the circulation around the cavity. In contrast, the circulation about the cusped cavity cannot be prescribed, but rather is a derived quantity. Finally, we state the condition for free detachment as

$$
\frac{d \omega}{d \zeta} \rightarrow 0 \quad \text { as } \quad \zeta \rightarrow 1 \quad \text { and/or } \quad \zeta \rightarrow-1
$$

whichever is applicable.

The above solution may be regarded either as for a direct problem with the prescribed quantities

$$
P[\hat{\beta}(s) ; \alpha ; \sigma ; S] \quad(0<s<S)
$$

or as for an "inverse problem" with the specification

$$
P^{\prime}\left[\beta(\xi) ; A_{1} ; \zeta_{0}\right] \quad(-1<\xi<1)
$$


in which $A_{1}=A / q_{c}$, the other parameters $\zeta_{c}, \zeta_{R}, \zeta_{J}$ being assumed already eliminated. One should note that the inverse problem is completely determined. On the other hand, in the direct problem, even with fixed detachments, $s(\xi)$ and $\beta(\xi)=\beta(s(\xi))$ are not known a priori. The right-hand sides of Equations (15) and (19) may be regarded as two nonlinear integral operators providing the functional transformations of $s(\xi)$ and $\zeta_{0}$ into the respective left-hand side members, $s(\xi)$ and $\zeta_{0}$ being the fixed points of the transformation.

Flat plate and symmetric wedges.-For these simple configurations, the direct problem immediately admits a unique explicit solution. Figure 5 is an example of the theoretical prediction by the open-wake model (Wu 1962) of the pressure distribution on both sides of a flat plate at several angles of attack; it is in very good agreement with the wind tunnel experiments of Fage \& Johansen (1927). Elsewhere one finds further evidence that the free-streamline theory generally provides results in satisfactory agreement with experiments, such as those of Meijer (1967), Parkin (1958), and Silberman (1959). Meijer presented detailed measurements of the pressure distribution on a flapped hydrofoil in a water tunnel, covering both the case when the near-wake is filled with water (at high cavitation numbers $\sigma$ ) and the case when the vapor-gas cavity is developed (at lower $\sigma$ ). These comparisons confirm the contention that the free-streamline theory is of comparable applicability in both wake and cavity flows.

As another point of comparison, the hydrodynamic forces and the cavity shapes given by the commonly used models are generally in close agreement for blunt bodies. For example, with the Riabouchinsky model the theoretical drag coefficient of a flat plate, held normal to the stream, has the following asymptotic expansion:

$$
C_{D}(\sigma)=C_{D}(0)\left[1+\sigma+\frac{\sigma^{2}}{8(\pi+4)}+O\left(\sigma^{4}\right)\right]
$$

where $C_{D}(0)=2 \pi /(\pi+4)=0.88$ is the classical Kirchhoff solution for $\sigma=0$. This $C_{D}(\sigma)$ agrees up to three terms with that of the reentrant-jet model, and is also in close accord with the result of the open-wake model for $\sigma$ less than 1 (in the last case, the factor $1 / 8$ of the term with $\sigma^{2}$ in Equation (23) is replaced by $1 / 6$ ). It should be stressed, however, that it is generally for thin bodies at small incidences that these theoretical models deviate from each other, especially at higher cavitation numbers, until the cavities become so short that the postulated flow configuration is no longer valid. Further, the degree of accuracy of the theoretical models in estimating the forces has been rather neglected in the past; this important issue will be discussed in Section 6 on the wall effects.

On the other hand, as $\sigma \rightarrow 0$ all these models reduce to the Kirchhoff 

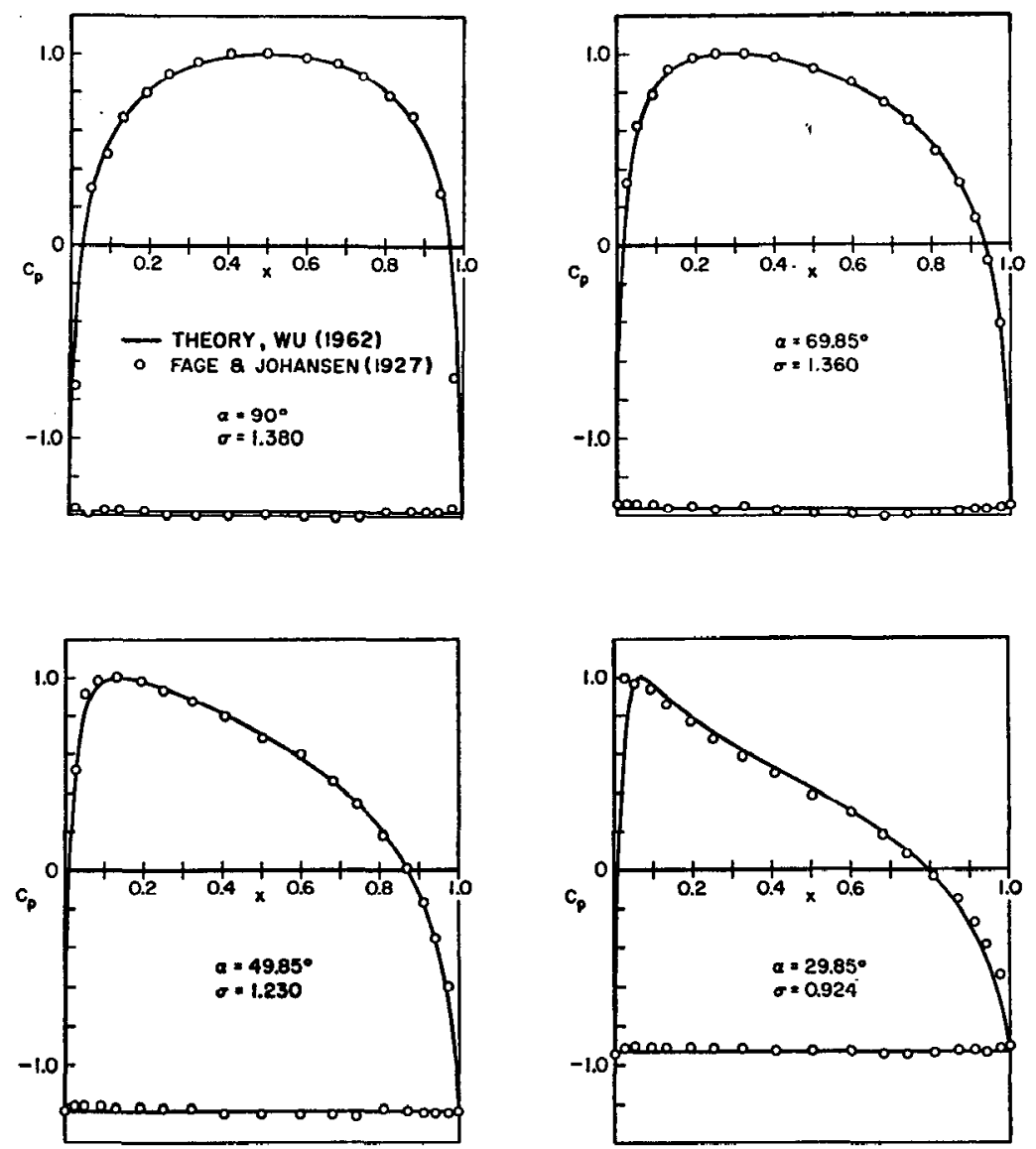

FIGURE 5. Pressure distributions on the wetted and separated sides of an oblique flat plate. Theory indicated by a solid line (Wu 1962); experiments indicated by a small circle (Fage \& Johansen 1927).

flow. In this limit, the asymptotic form of the free streamlines at large distances behind an arbitrary body is given by the following expression:

$$
y-y_{0} \sim \pm C_{1} x^{1 / 2}-C_{2} \log x+O\left(x^{-1 / 2} \log x\right)
$$

where $C_{1}, C_{2}$, and $y_{0}$ are constants depending on the body shape, the \pm signs referring to the upper and lower branch respectively. The first two coefficients $C_{1}$ and $C_{2}$ are related to the drag $D$ and lift $L$ according as

$$
D=\frac{\pi}{4} \rho U^{2} C_{1}^{2} \quad \text { and } \quad L=2 \pi \rho U^{2} C_{2}
$$


These are the outstanding features of the Kirchhoff flow at large distances.

Finally, we note that the existence of some explicit solutions for simple body shapes is of great significance in the study of existence and uniqueness theory and of numerical methods (to serve as an effective initial approximation with which to begin iteration) for general curved bodies.

Existence and uniqueness theory for curved bodies.-The conditions for the system of functional equations to possess a unique and meaningful solution to the free-boundary problem for curved bodies have been studied at length in the past fifty years. The theory for the problem of fixed detachment and, to a lesser extent, for the problem of free detachment is now well advanced, having inspired many original and important ideas in bringing about a brilliant chapter in mathematical analysis.

Apparently the first existence theorem for curved bodies was obtained by Nekrasoff (1922) for the Kirchhoff flow past circular arcs of small extent. His proof is constructive, based directly on iteration of the functional equation of the flow. After further extensions by his followers to include other body forms and flow models (Birkhoff \& Zarantonello 1957), the proof established the following Fixed-Point Theorem: For given $\hat{\beta}(s)$ and $\zeta_{0}$, if $A$ is sufficiently small, then the functional equation (15) can be solved by direct iteration, and the solution is unique.

A very general, powerful, but nonconstructive proof of existence of the infinite-cavity flow past a curved body, not necessarily of small curvature, was provided by Leray (1935) with application of the fixed-point theorem of Leray \& Schauder (1934). The detailed accounts of the proof and its further extensions can be found in Birkhoff \& Zarantonello (1957) and Gilbarg (1960).

Numerical methods.-By classical analysis, the solution of plane cavityflow problems is reduced to the determination of one unknown real function $s(\xi)$ and the parameters $A$ and $\xi_{0}$, which combined satisfy the nonlinear functional equations (15), (18), and (19) [an additional functional equation (20) is involved for each free detachment]. Even after this simplification, evaluations of these functional equations by current high-speed computers can still present a difficult computational problem.

Apparently the first calculations of free-boundary flows past curved bodies were performed by Brodetsky (1923), who computed the plane cavity flow past circular and elliptical arcs by a method of trigonometric interpolation. This method was later extended (Birkhoff \& Zarantonello 1957) by using a truncated series representation of the function $\Omega=\Gamma(\xi)+i \beta(\xi)$ [see Equation (16)], the coefficients being computed by a process of averaged iteration.

Recently an integral iteration method, which is essentially of the Picard type, was developed by Wu \& Wang (1964a) for computing the functional equations (15) and (19). This method was subsequently modified (Wu \& Wang 1964b) to give an approximate numerical scheme based on iteration 
of the function $s(\xi)$ and the parameter $\xi_{0}$ on different levels, at a great saving of computing steps while at the same time retaining a high degree of accuracy. Success of this method depends on a proper choice of the initial approximate solution (generally an inclined flat plate, or a plate with a flap at a suitable incidence) and on an adequate execution of averaged iteration to ensure that each iterated solution is meaningful (such as $\zeta_{0}$ always remaining in the flow region). This method has been applied successfully to several cavitating hydrofoil problems (Wu 1968). The method has also been successfully applied to cavitating hydrofoils having rounded noses (Furuya \& Acosta 1972) with a fixed separation point near the leading edge. Again, numerical convergence is satisfactory provided that a proper averaging of the successive iterations is followed, although more iterations for this type of problem are required.

\section{Axisymmetric and Three-Dimensional Gavity Flows}

Efforts made in solving three-dimensional cavity and wake problems have been confined largely to the numerical approach, or at best seminumerical (relying on analysis to reduce the problem to a simpler form), since the complex function theory, which proves to be a powerful tool for plane flows, cannot be fully utilized here. Because of the great mathematical difficulties, there is still no known exact solution of three-dimensional bodycavity problems, though several approximate methods have proved to be useful.

Surface-singularity method.-Trefftz (1916) made the first major efforts to calculate axisymmetric jets based on an exact theory. His integral equation method, which was for years the principal contribution to the axisymmetric free boundary problems, is actually very general. In terms of the cylindrical polar coordinates $(x, r, \theta)$, with $x$ pointing in the free stream direction, the velocity potential $\phi(x, r)$ satisfies the equation

$$
\phi_{x x}+\phi_{r r}+\frac{1}{r} \phi_{r}=0
$$

With the theoretical model chosen, the boundary conditions become

$$
\begin{array}{cccc}
\frac{\partial \phi}{\partial n}=0 \quad \text { on } & \Gamma_{1}+\Gamma_{2} \\
\frac{\partial \phi}{\partial s}=q_{c} & \text { on } \quad \Gamma_{2}
\end{array}
$$

where $\Gamma_{1}$ and $\Gamma_{2}$ denote the curves in the meridian cross section of the flow intercepted by the body and cavity boundaries, respectively, $\mathrm{n}$ is a unit normal vector, inward to flow, and $s$ is a unit tangential vector at $\Gamma_{1}+\Gamma_{2}$. 
Using Green's theorem for the axisymmetric potential $\phi(x, r)$, Trefftz derived the following integral equation for $\phi$ :

$$
\begin{aligned}
\phi(s) & =\int_{\Gamma_{1}+\Gamma_{2}} G(s, t) \phi(t) d t+f(s) \quad\left(s \text { on } \Gamma_{1}+\Gamma_{2}\right) \\
G(s, t) & =r(t) \frac{\partial \Phi(s, t)}{\partial n_{t}}, \quad \Phi(s, t)=\frac{2}{\pi r_{1}} K(k) \\
r_{1}{ }^{2} & =\left(x_{s}-x_{t}\right)^{2}+\left(r_{s}+r_{t}\right)^{2}, \quad k^{2}=\frac{4 r_{s} r_{t}}{r_{1}^{2}}
\end{aligned}
$$

$\mathbf{x}_{t}=\left(x_{t}, r_{s}\right), \mathbf{x}_{t}=\left(x_{t}, r_{t}\right)$ being the position vectors of two points on $\Gamma_{1}+\Gamma_{2}$, $r(t) \equiv r_{t}, K(k)$ denoting the complete elliptical integral of the first kind, and $f(s)$ standing for a fixed function of the arc length $s$. Here, $\Phi(s, t)$ is the potential at $s$ due to a ring source at $x=x_{t}$ and $r=r_{t}$. The problem at hand is to determine the shape of $\Gamma_{2}$, and hence implicitly the kernel $G(s, t)$, so that $\phi$ satisfies condition (28) and the integral equation (29). Treff tz computed $\phi(s)$ for a jet emanating from a circular orifice in a plane by a scheme of successive approximations, and found the jet contraction coefficient $C_{c}$ to satisfy $0.60<C_{c}<0.64$. Regarding the numerical method, Gilbarg (1960) commented that extreme refinement, near detachment in particular, is essential to accuracy. An interesting feature of this method is that calculations of the flow field are reduced to the boundary of one dimension, much the same as the integral equation method for plane flows.

From a similar equation Levinson (1946) determined the asymptotic shape of infinite axisymmetric cavity as

$$
r(s)=C x^{1 / 2}(\log x)^{-1 / 4}\left[1-\frac{\log \log x}{8 \log x}+O(\log x)^{-1}\right]
$$

and showed that the cavity drag $D$ is related to the shape factor $C$ by

$$
D=\frac{1}{8} \pi \rho U^{2} C^{4}
$$

Thus, in comparison with Equation (24), the asymptotic shape of axisymmetric cavities is narrower than that of the plane case by the factor $(\log x)^{-1 / 4}$.

The method of distributing singularities over the body and cavity surfaces has been applied to different problems (Shiffman \& Spencer 1951, Salamotov 1959). Recently this method was extended by Struck (1970) to include axisymmetric bodies at small incidences by allowing the strength of annular sources to vary with the azimuth. Because he used the open-wake model, the integral equation derived by Struck for the axisymmetric component is very similar to Trefftz's equation, while the cross-flow component 
is linearized. The free boundary was determined by an iteration, starting with an assumed location. The integral equation was computed by the Gaussian quadrature for the circular disk and cones over a range of the incidence, and for the sphere; the results of pressure distribution and free-surface shape were compared with known experiments. At $\sigma=0$ (infinite cavity) his drag coefficient of the disk satisfies $0.824<C_{D}<0.829$, and for the sphere, $C_{D}(0)$ $=0.31$, the corresponding free-detachment point being at $\theta_{s}=57^{\circ}$ from the forward stagnation of the sphere.

Stream-function method.-In axisymmetric flows the stream function $\psi(x, r)$ satisfies the equation

$$
\psi_{x x}+\psi_{r r}-\frac{1}{r} \psi_{r}=0
$$

An ingenious method was developed by Garabedian (1956), who considered the axisymmetric flow in a space of $(\epsilon+2)$ dimensions and calculated its stream function $\psi(x, r ; \epsilon)$ [which satisfies an equation similar to Equation (34), only with the coefficient $1 / r$ replaced by $\epsilon / r]$ by expanding it about the corresponding plane flow $\psi_{0}(x, y)=\psi(x, r ; 0)$, where $y \equiv r$, in a power series in $\delta=\epsilon /(\epsilon+2)$. The required solution in the physical case is then $\psi(x, r ; 1)$ with $\delta=1 / 3$. His drag coefficient of the circular disk in infinite cavity flow is $C_{D}=0.827$.

It is also possible to make use of Equation (34) when one chooses to represent the axisymmetric body-cavity boundary by a vortex sheet composed of elemental vortex rings. In this formulation $\psi(x, r)$ assumes an integral representation involving the unknown strength of the vortex sheet. Application of the boundary conditions on the body $(\psi=0)$ and cavity boundary $\psi=0$, $\left.\psi_{n}=q_{c} r\right)$ then leads to the desired integral equation for $\psi$.

The relaxation method.-Southwell \& Vaisey (1946) first applied the relaxation method to a number of free-surface flow problems, including the cusped cavity behind a cylinder and sphere; the axisymmetric jet from an orifice; a two-dimensional planing surface under gravity; and a few other motions. This original contribution has been followed by other studies using this method.

A recent development is the contribution by Brennen (1969b), who obtained a numerical solution of the axisymmetric flows with a finite cavity past a circular disk and sphere, together with some new experimental results. In his theory the Riabouchinsky model is adopted for its desirable symmetry, and the basic equations take the velocity potential $\phi$ and stream function $\psi$ as the independent variables. First, by interchanging the dependent and independent variables,

$$
\frac{u}{q^{2}}=r r_{\psi}=x_{\phi} \quad \text { and } \quad \frac{v}{q^{2}}=r_{\phi}=-r x_{\psi}
$$


where $q^{2}=u^{2}+v^{2},(u, v)$ being the $(x, r)$-component velocities. Then upon elimination, there results for $f \equiv r^{2}$ the equation

$$
\frac{\partial^{2} \log f}{\partial \phi^{2}}+\frac{\partial^{2} f}{\partial \psi^{2}}=0
$$

which is due to Woods (1951). The boundary conditions are

$$
\begin{aligned}
\theta(\phi, 0) & =\tan ^{-1} \frac{f_{\phi}}{f^{1 / 2} f_{\psi}} & & \text { on } \Gamma_{1} \\
\frac{4}{q_{c}^{2}} & =\frac{f_{\phi}^{2}}{f}+f_{\psi}^{2} & & \text { on } \Gamma_{2}
\end{aligned}
$$

where $\theta=\tan ^{-1}(v / u)$ is the flow angle and $\Gamma_{1}$ and $\Gamma_{2}$ have the same significance as before. The nonlinearity of Equation (36) is of little disadvantage in the numerical method; it is far outweighed by the advantage gained by the known straight flow boundaries in the $(\phi, \psi)$ plane. Brennen applied the relaxation calculations to Equations (36)-(38) in a quadrant of the flow (owing to the symmetry) bounded laterally by concentric cylinders of various sizes, the unbounded-flow case being reached by extrapolation. Consequently the results also furnished useful information about the wall effect in the axisymmetric case. In these calculations the singularities at stagnation and detachment and treatment of the flow along the free boundary (on the axis $\psi=0$ ) require proper handling, as is common to other numerical methods. The maximum error in the numerical results of $f$ was estimated to be less than 0.5 percent. Comparison with the experiments shows that the theory is very satisfactory in the disk case; Brennen's value of the drag coefficient at $\sigma=0$ is $C_{D}=0.826$, which is in agreement with Garabedian's 0.827 . For the sphere with an infinite cavity, Brennen found a value of $C_{D}=0.317$, and detachment at an angle of about $\theta_{s}=56^{\circ}$ (by extrapolation), which are in close agreement with Struck's results. These consistent theoretical predictions, however, are at variance with the experimental observations. The measurements of the sphere drag by Eisenberg \& Pond (1948) and Hsu \& Perry (1954), when extrapolated to $\sigma=0$, yield a mean of $C_{D} \simeq 0.25$; and also indicate that the measured $C_{D}(\sigma)$ increases with $\sigma$ at a rate considerably greater than that predicted by the theory. The discrepancy between the measured separation position and the predicted detachment appears to be even worse. The experimental studies by Hsu \& Perry (1954) and Brennen (1969b) show that the observed separation position on a cavitating sphere depends on both cavitation number $\sigma$ and Reynolds number $R$. Invariably its position lies downstream of that predicted by inviscid theory, the difference increasing as $R$ is reduced. At $\sigma=0.1, R=10^{5}$, for instance, the (laminar) separation position is at an angle $\theta_{8} \simeq 82^{\circ}$, as against the theoretical value of $\theta_{\mathrm{s}} \simeq 58^{\circ}$. The results from wind tunnel tests are essentially the same (Carmody 1964). These evidences all indicate that the viscous effect on the position of free separation from curved bodies, and hence on the resistance 
of fluid, is of considerable importance. They also cast light on the question of whether some modification of the Brillouin-Villat condition for free detachment will be necessary and sufficient to improve the accuracy of inviscid theory. There is very little published work on this viscous effect, but Struck (1970) did apply boundary-layer theory to evaluate the velocity profile on a sphere, and to determine the separation point by iteration, the pressure distribution used in computation being supplied experimentally. In a specific case, his iterated procedure predicted a viscous separation at $\theta_{\mathrm{q}} \simeq 77^{\circ}$, while the measured value was $\theta_{8} \simeq 81^{\circ}$. This already shows considerable improvement.

Approximate methods. - A few approximate methods, though based on less refined considerations, have served useful purposes. Various methods have been proposed to correlate plane free-boundary flows with corresponding axisymmetric cases. They are based on a correspondence principle which assigns the same pressure distribution to an obstacle in axisymmetric flow as in the corresponding plane flow. This principle was applied by Plesset \& Shaffer (1948) to the cone and wedge of the same angle in a Riabouchinsky flow at the same cavitation number, $\sigma$; their value of $C_{D}$ of the disk at $\sigma=0$ is 0.805. Different refinements were introduced by Fisher (1944) and Armstrong \& Dunham (1953); they gave comparable results of $C_{D}(0) \simeq 0.825$ for the disk. The good agreement of these approximate solutions with the exact theory and observation becomes less satisfactory, however, for cones of smaller angles. In this case Armstrong (1954) suggested further improvement by correcting the velocity gradient near the vertex.

Three-dimensional cavities. - In the category of three-dimensional cavity and wake flows without axial symmetry, only a very few aspects of the general problem have been investigated. A class of cavity flows past a slender plate held at a small incidence was studied theoretically by Cumberbatch \& Wu (1961). Based on slender-body theory, the flow is analyzed in two parts: a longitudinal component that accounts for the variation of the cavity cross section, and a cross flow that is evaluated with the Riabouchinsky model. We have already mentioned the theory of Struck (1970) for an axisymmetric body at a small incidence to flow, with only its base open to the cavity or wake. At the other extreme, the problem of a cavitating hydrofoil of large aspect ratio was treated by Cumberbatch (1961), based on a linearized theory and the lifting-line idea. Another interesting problem of large aspectratio cavities was discussed by Benjamin (1964), who considered the matching of the near- and far-field solutions through a singular perturbation method.

\section{Wall Effects in Cavity Flows: A Critical Examination of Flow Models}

The problem of wall effects in cavity flows is of practical importance in interpreting water-tunnel experiments. It is also of theoretical interest since 
the presence of lateral wall constraints on the flow can provide a crucial condition under which the accuracy of different flow models can be examined by comparison with experiment. The wall effects in cavity flows are invariably more appreciable than those in wind-tunnel and water-tunnel tests of bodies without wake or cavity formation, since the flow near the solid body is affected conjointly by the wall constraints and by the changes in cavity shape as the wall-spacing or the cavitation number is varied. Because of the mathematical difficulties, the earlier theoretical studies were largely limited to the choked flow case (when the confined cavity becomes infinite in length) and to simple body forms. Several attempts were made previously to treat finite cavities, using either the Riabouchinsky or the reentrant-jet model, again with simple body shapes in plane flows. An expository survey of these historical developments has been given by $\mathrm{Wu}$, Whitney \& Brennen (1971), and will not be repeated here.

In this recent study by $\mathrm{Wu}$, Whitney and Brennen, the three theoretical models in common use (the Riabouchinsky, open-wake, and reentrant-jet models) were employed to investigate the effect of solid tunnel walls upon the inviscid, nonlifting plane flows past symmetrical bodies of arbitrary shape. As in the unbounded flow case, the solution for curved bodies was given in the form of functional equations, which can be solved only by numerical methods. For symmetric wedges, however, it was possible to carry out an asymptotic expansion of the in tegral solution for small to moderate ratios $\lambda$ of the body frontal width to tunnel spacing.

For the Riabouchinsky model, this asymptotic expansion reduces the drag coefficient of wedges to the following simple formula:

$$
\begin{gathered}
\frac{C_{D}(\sigma, \lambda)}{1+\sigma}=\frac{C_{D}\left(\sigma^{\prime}, 0\right)}{1+\sigma^{\prime}}+O\left(\lambda^{2}\right) \\
\sigma^{\prime}=\frac{2}{3} \sigma+\frac{1}{3} \sigma^{\prime \prime}
\end{gathered}
$$

where $\sigma^{\prime \prime}=\left(p_{b}-p_{c}\right) / \frac{1}{2} \rho V^{2}$ is a new cavitation number based on the minimum pressure $p_{b}$ and the maximum velocity $V$ on the tunnel wall (that is, at a point opposite maximum cavity width). Equations (39) and (40) provide a simple and practical wall-correction rule, which takes a "measured" drag coefficient $C_{D}(\sigma, \lambda)$ of a wedge, at given cavitation number $\sigma$ and tunnelspacing ratio $\lambda$, and converts it to an estimated drag coefficient $C_{D}\left(\sigma^{\prime}, 0\right)$ for the same wedge in unbounded flow $(\lambda=0)$, but at a different cavitation number, $\sigma^{\prime}$. The new cavitation number $\sigma^{\prime}$ can be found either by actual measurement of both $\sigma$ and $\sigma^{\prime \prime}$, or by theoretical calculations. The accuracy of the above wall-correction rule, in comparison to the exact values of $C_{D}(\sigma, \lambda)$ computed from the original theory, was found to be excellent for all wedge angles, for $\lambda$ up to $1 / 6$, and for $\sigma$ less than 1 , a range that well covers practical interest.

For the open-wake model, the asymptotic expansion of the theoretical 
solution also yields a simple formula in which the drag coefficients $C_{D}(\sigma, \lambda)$ and $C_{D}\left(\sigma^{\prime}, 0\right)$ are again related, up to order $O(\lambda)$ by Equation (39), except that $\sigma^{\prime}$ is now given by

$$
\sigma^{\prime}=\sigma-\left(1+\sigma^{-1}\right) C_{D}(\sigma, \lambda) \lambda+O\left(\lambda^{2}\right)
$$

Thus, with the open-wake model, Equations (39) and (41) play the same role as Equations (39) and (40) did for the Riabouchinsky model; they provide a two-way correction from the $C_{D}(\sigma, \lambda)$ of the bounded flow to the $C_{D}\left(\sigma^{\prime}, 0\right)$ of the unbounded flow and vice-versa. These two wall-correction rules both were found to be very accurate representations of their respective exact solutions. Their difference, which is negligible for large wedge angles and increases to an appreciable extent as wedge angle is reduced, therefore provides a direct comparison between the two theoretical models.

A series of experiments in a horizontal closed water tunnel was designed specifically for the purpose of conducting a critical examination of the accuracy of the flow models in representing physical flows. Proper consideration was also given to the viscous effects due to the boundary layer at the tunnel walls in causing a "horizontal buoyancy"; to the skin-friction increment at the wedge surface; and to the wake blocking. These effects proved to be only secondary; their corrections were nevertheless taken into account. A typical final result is given in Figure 6 for a wedge of total vertex angle of $30^{\circ}$. The experimental drag coefficients were obtained both by pressure integration and by direct balance measurcment, the results by the later method showing greater scatter. The theoretical results determined from the Riabouchinsky model, as shown in Figure 6, are the closest of the chosen models to the experimental measurements over a range of $\lambda$ up to values as high as $\lambda=0.236$. The open-wake model was found to overestimate the drag coefficient (by 10 percent, as is typical in the range of this case). Further, the pressure-integrated drag data (in open symbols) were corrected for wall effects using Equations (39) and (40); the results are the solid points in Figure 6, which all lie very close to the unbounded-flow theoretical line. Of the salient features of the wall effects we note that the wall-constraint results in lower $C_{D}$, at the same cavitation number, than in the unbounded-case, and this percentage drag reduction increases with decreasing wedge angle!

In summary, for the pure drag case the essential finding is that, of the theoretical models, that due to Riabouchinsky is superior throughout the range tested. The accuracy of the correction rule based on that model has also been firmly established. This correction rule, though only tested for wedges, may possess a wider validity, since the body geometry is only implicitly involved in the correction formula.

In the case of lifting flows, the theoretical study by $\mathrm{Wu}$, Whitney \& Lin (1969) showed that the wall effects on both lift and drag are generally less significant than in the pure drag case. In general, the boundary effects on the forces of a body in a free jet are also relatively small. 


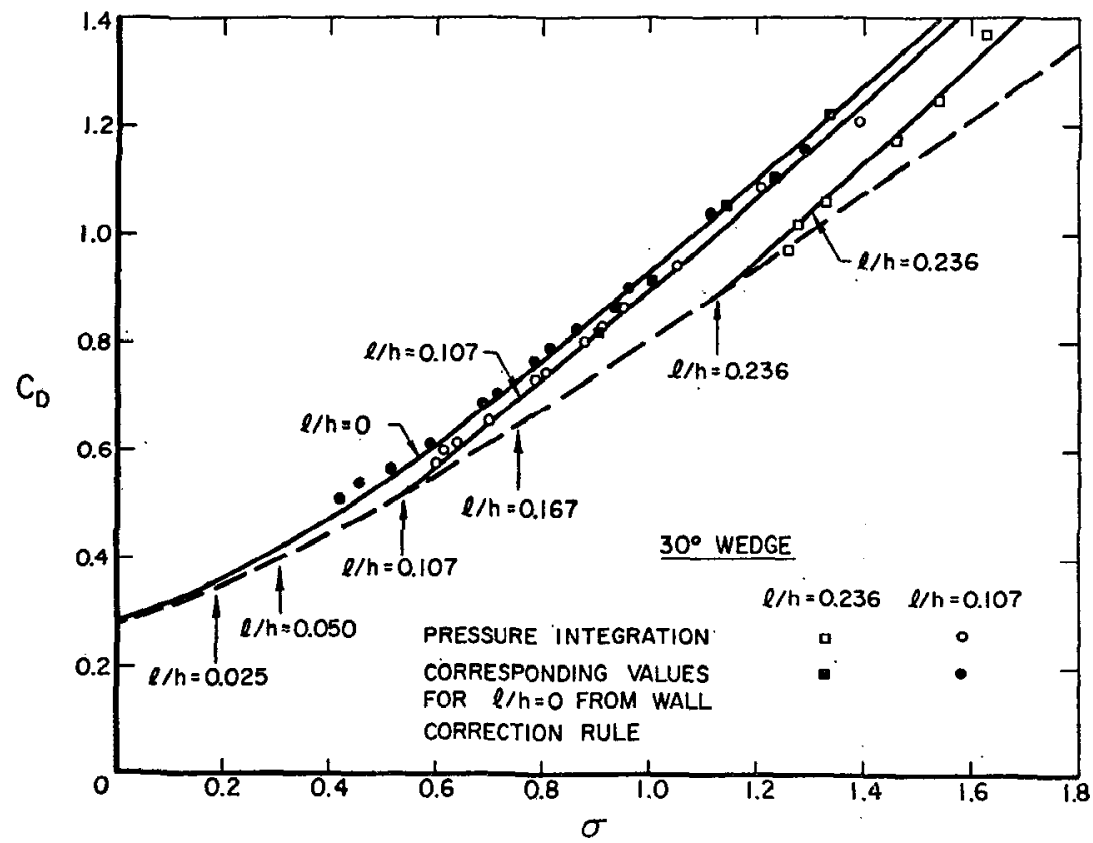

FIGURE 6. Drag of a $30^{\circ}$ wedge cavitated in a closed water tunnel ( $\mathrm{Wu}$, Whitney \& Brennen 1971). Theory: Riabouchinsky model ——; cholsed-flow line, -..-; $\ell / h=$ wedge-base width/tunnel spacing.

\section{Gravity Effect}

The effect of gravity arises in cavity and jet flows involving a free boundary that separates a heavy fluid from a lighter one; it modifies the condition of constant pressure [Equation (5) for the gravity-free case] on a free boundary, in a gravitational field $\mathrm{g}$, to

$$
\phi_{t}+\frac{1}{2}(\nabla \phi)^{2}-\mathbf{g} \cdot \mathbf{x}=\frac{1}{2} U^{2}(1+\sigma) \quad \text { on } F(\mathbf{x}, t)=0
$$

provided the inertial effect of the lighter fluid is again neglected, and the definition of cavitation number, $\sigma$, is suitably modified. Thus, even in the case of steady flows $\left(\phi_{t}=0\right)$, a free surface of constant pressure is no longer a surface of constant speed. The early studies of these more difficult problems generally made use of inverse methods for steady, plane flows. These methods are based on the idea that the free streamline $F=(x(\phi), y(\phi))$ is an analytic curve [proved independently by Gerber (1951) and Lewy (1952)], so that both this curve $F$ and Equation (42) can be continued analytically into the flow and some specific relation between them used to generate a physical flow. For surveys of literature in this category we refer to Birkhoff \& Zarantonello (1957) and Gilbarg (1960). 
For explicit determination of flows past given boundaries in a gravity field, a different approach is based on extension of the integral-equation method already described for the gravity-free case. This idea may be traced back to the original contribution by Joukowsky (1890). A similar method was developed by Richardson (1920), who presented two types of integral equations for free-surface flows with gravity. These two formulas have been employed by Richardson and others as semi-inverse methods for the determination of exact solutions to several specific problems. Although these problems may seem somewhat artificial, a recent application of the method by Yih (1960) for a number of special cases yielded results of considerable interest. In general, however, integration of the functional equation for given body shape still encounters great mathematical difficulties.

A fundamental difficulty may be ascribed to the presence of a free boundary of unbounded extent, such as in the problem of a planing surface at, or a cavitating body moving underneath, an infinite water surface. Near such a free boundary, a measure of the relative importance of the gravity and inertial effects is given by the Froude number, $F r=U /(g \ell)^{1 / 2}$, where $U$ is a characteristic velocity and $\ell$ a characteristic length. The asymptotic approximation (in the Poincaré sense) of the solution for large values of $\mathrm{Fr}$ (which is typical in cavity and jet flows) can be seen to have certain nonuniform behavior. In fact, since the inertial effect due to an isolated source or vortex falls off like $U^{2} / r$ at large distances $r$, the relative magnitude of the gravity effect to the local inertial effect is

$$
\frac{\text { gravity effect }}{\text { inertial effect }} \simeq \frac{g}{U^{2} / r}=\frac{r}{\ell} \frac{1}{F r^{2}}
$$

This ratio tends to zero as $F r \rightarrow \infty$, with $r / \ell$ fixed; but it grows beyond all bounds as $r / \ell \rightarrow \infty$, for fixed $F r$, no matter how large. This nonuniform behavior of the "local Froude number" is very much analogous to the Whitehead paradox associated with the Stokes flow at low Reynolds numbers. (Conversely, for Fr small, a similar argument can be given to indicate that the gravity effect is predominant, almost everywhere, except in a region where the curvature of streamlines is large.) It is therefore clear that when the solution is sought by expansion for large or small $F r$, the method of matched asymptotic expansion is useful. Following this approach, Rispin (1967) and Wu (1967) have developed a singular perturbation theory for nonlinear free-surface flow problems for two-dimensional planing surfaces; it was recently applied by Dagan (1971) for a submerged cylinder. Using the same method, Shen \& Ogilvie (1972) evaluated a nonlinear theory for finitespan planing surfaces and discussed the "dimensionality effect."

Based on the foregoing argument, a uniformly valid solution can be obtained by using the regular perturbation method, for $\mathrm{Fr}$ large, if the free boundary occurring in a flow is only finite in extent, such as a finite cavity in an unbounded flow. This method was indeed put to good use by Parkin 
(1957a), Acosta (1958), and Larock \& Street (1967), in treating the gravity effect in cavity flows.

\section{Comphessibility EFfect}

As the theory of compressible jets is a well developed part of gas dynamics, and has been well covered in the literature, we shall present only an outline of the methods and discuss their relations with the corresponding incompressible flows. The first fundamental contribution on the subject was made by Chaplygin (1904), who, in his celebrated memoir, derived for the stream function of a steady, irrotational, and isentropic plane motion an exact equation, which is linear in the hodograph variables and valid for subsonic as well as supersonic speeds. Chaplygin also furnished an exact method of solution by separation of variables, and further established a correspondence principle which relates solutions of analogous problems of compressible and incompressible flows. Applications of this principle to various types of compressible free-surface flows have appeared in the literature.

For a polytropic flow past a prescribed obstacle, the Chaplygin equation can be converted to a system of integral equations by a procedure similar to the derivation of the Villat functional equation for the incompressible case (see Birkhoff \& Zarantonello 1957, p. 196). Mackie (1958) used this method to obtain an exact solution for a symmetric wedge with an open wake in a polytropic flow, the resulting integral equation being solved by the Sherman inversion formula.

An approximate method that reduces the flow problem to a conformal mapping between the complex potential $f=\varphi+i \psi$ and the hodograph variable $\omega=\tau+i \theta$ (both being defined as proper to compressible flows) was developed by Chaplygin (1904) and later by Tsien (1939) with the introduction of a hypothetical pressure-density relation for the gas,

$$
p=a-\frac{b}{\rho}
$$

which may be regarded as an approximation of the actual isentropic curve $p=p(1 / \rho)$ by its tangent at a point $\rho=\rho_{1}, p=p_{1}$, thereby determining the coefficients $a, b$. We have a Chaplygin gas if the point $\left(1 / \rho_{1}, p_{1}\right)$ is referred to the stagnation point, and a Kármán-Tsien gas if it is referred to the point at infinity. So much facility is gained by this approximation that practically any compressible flow problem can be resolved once the solution to its analogous incompressible case is explicitly known. Chaplygin demonstrated this procedure in his treatment of a compressible flow past an inclined plate with an infinite wake; his solution indicates an increase in the normal force on the plate due to the compressibility effect, and reduces to Rayleigh's solution in the incompressible limit. Various other problems of subsonic free surface flows have been treated by this method or some variation (Jacob 1946, Oudart 1949, Pai 1954, Bergman 1955). 
This approximate method can be extended also to supersonic flows; the resulting equations are of the hyperbolic type, and have been solved in different cases by Coburn (1945) and Chuang (1955). In some instances, the linear theory is sufficient to yield important features of the flow, as demonstrated by Prandtl $(1904 \mathrm{~b}, 1907)$ in his elegant analysis of supersonic jets. For the mixed-type flows, Sedov $(1949,1966)$ gave an equation covering both subsonic and supersonic speeds, and suggested an approximate relationship between $p$ and $1 / \rho$ in the form of a bent line with a vertex at the sonic point. This affords improvement in comparison to the method of using Tricomi's transonic equation, which provides good accuracy only in the region of the sonic point. Several specific jet and wake bubble problems in the transonic range have been considered by Frankl (1945), Skripkin (1955), Sakurai (1956), Helliwell \& Mackie (1957), and Fisher (1963).

For supersonic and hypersonic wakes, the flow angle and pressure at the outer edge of the wake shear layer can be determined locally (by the CroccoLees theory), this feature being in sharp contrast to subsonic wakes, where they depend on the entire flow. Further, the viscous shear in the wake becomes increasingly more pronounced at higher speeds. With this physical background, an integral-moment method, derived by taking integral moments of the boundary-layer equations, has been actively developed in the past decade, notably by Lees and co-workers (Lees \& Kubota 1957, Lees \& Reeves 1964, Reeves \& Lees 1965, Grange et al 1967). At present, this is the method that is both rational and useful for engineering problems though various numerical methods have also been adopted.

\section{Unsteady Cavity Flows}

The development of the theory of unsteady cavity flows and jets is relatively more recent. By unsteady flows we mean the time-dependent motions that can be generated by an unsteady body movement, by a temporal and spatial variation in the free stream, and by changes in the cavity pressure through forced ventilations, while the spontaneous unsteadiness due to instability of flow and turbulent mixing is not included in the present consideration. These unsteady-flow problems are in general extremely difficult for several rather obvious reasons. First, the location of an unsteady free boundary becomes totally unknown, even in the potential or the hodograph plane. Unlike the steady case, the unsteady surface of constant pressure is no longer a surface of constant speed, nor is it a streamline-it is a material line [see Equations (4) and (5)]. Further, these unsteady flows retain a "memory" of their history through the explicit dependence on time-rate of changes in both the kinematics and dynamics of the free boundary. Another difficulty arises when the cavity volume is permitted to vary with time; this additional degree of freedom requires a definite physical mechanism, and hence a theoretical model, in the formulation of the problem and this has been a disputed issue for sometime. Largely owing to these difficulties, only 
mild success has been achieved for a small number of cases in this general subject.

A particular class of problems, initiated by the work of von Kármán (1949), is concerned with accelerating flows or accelerating bodies with a permanent cavity shape. According to Equation (5) (an additional term of the D'Alembert pressure is to be inserted in the case of accelerating bodies, if referred to the body frame), this class of flows is characterized by a velocity potential $\phi(x, y, t)=U(t) \phi_{1}(x, y)$, with $U$ satisfying $a / U^{2}=$ const, $a \equiv d U / d t$. Von Kármán considered an accelerating flow normal to a flat plate held fixed in an inertial frame, and found a solution for a particular Froude number $U^{2} / a h$ ( $h$ being the plate half-width) at which the permanent cavity closes with a rear stagnation. This case was extended by Gilbarg (1952) to polygonal obstacles and to all possible values of $U^{2} / a h$. Yih (1960) emphasized the importance of the reference frame and considered both the case of an accelerating flow past a body at rest and the case of a body accelerating through an otherwise stationary fluid, his solution including von Kármán's as a special case. This class of flows, albeit rather artificial, uses familiar methods and provides a first step towards understanding the more difficult problems. In the general case, we must of course admit the freedom for cavities to vary with time.

It follows that the mathematical formulation of flows with cavities whose shape and/or volume are changing with time, deserves particular attention. For the case of small amplitude movements of a cavity boundary from its mean position, Gilbarg (1952) first proposed that the material line defining the unsteady cavity boundary may be approximated by the streamline of its mean position, and argued this would be valid for slow rates of change. This approximation was adopted by Woods $(1954,1955,1957)$ in treating some specific Riabouchinsky flows, infinity cavities, and oscillating stalled airfoils. It was used also by Parkin (1957b), Wu (1957b), Timman (1958), and Geurst (1960) in the linearized theory for unsteady cavity flow past thin bodies. We must note, however, that Gilbarg's approximation is too crude to describe the essential dynamic behavior of cavity boundaries in unsteady motion, as can be shown by a simple test with elementary water waves. For a perturbation theory to be consistent, approximations to the kinematic and dynamic free-surface conditions [Equations (4) and (5)] must be carried to the same degree of accuracy. Indeed, a correct formulation of the free-boundary conditions was given as early as 1880 by Lord Kelvin for a vibrating columnar vortex, and later by Ablow \& Hayes (1951), in an unpublished work, for perturbed jets and cavity flows.

As regards changes in the volume of a vapor-gas cavity, continuity requires that the exterior liquid must flow outward or inward across a fixed control surface enclosing the cavity at the same rate as the variation of cavity volume, since liquid compressibility is usually negligible and any phase change will involve large changes in density. In practice, the liquid medium usually has a free surface at some distance away, and variations 
of cavity volume are made possible by exposing the free surface to a fixed or variable pressure. On this physical basis $\mathrm{Wu}(1958)$ proposed that the volume change of an unsteady cavity can be formulated by introducing a timedependent source at infinity, the strength of which can be either arbitrarily prescribed or determined in specific cases. Although an unsteady source in plane flows generates a pressure that is logarithmically singular at infinity, corresponding to a source of infinite energy, such an objectionable situation would not arise in reality since the flow is never two-dimensional in the large. This physical picture has been elucidated by Benjamin (1964), who showed mathematically the equivalence between the hypothetical plane flow and the inner region of a real three-dimensional flow with small spanwise variations. This model of unsteady cavities has been applied by Wang \& Wu (1963) and Woods (1964) for growing cavities behind hydrofoils. In this phase of development, still other theoretical models were proposed by Timman (1958), Birkhoff (1950), and Tulin (1958). Timman introduced a distribution of sources and sinks, representing growing or collapsing small bubbles, in the foamy wake of a finite cavity. Birkhoff, and independently Tulin, proposed a model that depends on compressibility of the liquid medium to form shock waves as a means of matching the variation in cavity volume. It is thought that the compressibility of the foamy wake is generally too small, and the energy required for shock formation in liquid is too large to describe the actual phenomenon.

A general class of unsteady cavity flows that seems to offer promise for further theoretical studies is the case in which the unsteady component of the motion is a small perturbation of a steady cavity flow already established. For this type of flow a perturbation theory has been developed by Lord Kelvin (1880), Ablow \& Hayes (1951), Fox \& Morgan (1954), and Curle (1956). Subsequently a more general formulation of the theory was given by Wang \& Wu (1965), including further considerations on (i) an appropriate condition for varying cavity volume, and (ii) the radiation condition for surface waves on a cavity boundary. The surface waves resulting from unsteady disturbances propagate downstream along the cavity boundary, in much the same way as ordinary gravity waves in water except that the centrifugal acceleration due to the curved basic flow acts as the local restoring force. These dynamic waves degenerate to kinematic waves when Gilbarg's approximation is used. Some recent progress towards further development of the theory was made by Wang (1970) and Kuznetsov et al (1971).

The perturbation theory of unsteady motion also facilitates investigations of the hydrodynamic stability of a given stationary free-surface flow, in the absence of extraneous disturbances, as an eigenvalue problem. Using this approach, Fox \& Morgan (1954) considered the stability of the firstorder perturbations of several inviscid flows, and obtained the following results. (i) For a symmetric jet of finite width impinging normally upon a finite plate, the flow is neutrally stable or stable. (ii) For two equal and opposite plane jets impinging upon each other, there exist unstable perturba- 
tions that are symmetric about the outgoing axis of symmetry, whereas all other admissible perturbations are stable. (iii) For a jet issuing from a container bounded by two plane plates subtending an inner angle $2 \pi / n, n=2^{m}$, $m=0,1,2, \cdots$, all the perturbations are stable except when $n=1$ (the Borda mouthpiece), in which case there exists an isolated unstable mode.In conclusion, it must be pointed out that the physical effects of viscosity, gravity, and surface tension, which were left out in the above treatment, may also be of primary importance to the question of stability. The evidences are ample, as we have observed in Section 2 on the physical background of real flows.

While the theoretical developments of unsteady cavity flow have met great mathematical difficulties, the experimental techniques for these studies are also by no means simple. Briefly, we note that the presence of unsteady elastic forces in test bodies generally makes accurate measurements of the hydrodynamic forces very difficult because these two types of forces can both be significant in response to the large virtual masses of the unsteady liquid stream, and the high speed of propagation of elastic waves can cause multiple reflections in the solid. Moreover, the unsteadiness in the flow usually extends quite far from an oscillating body tested in water tunnels; it may further excite vibrations of the tunnel boundaries and affect the dynamics of the entire system. Some experimental investigations of oscillating, cavitated hydrofoils were conducted by Meijer (1959), DeLong \& Acosta (1969), and Klose \& Acosta (1969). In some instances, forced ventilation with air was employed to maintain the cavity. Generally the experimental results were in reasonable agreement with the theoretical estimates at small angles of attack; systematic departures were observed as the angles became larger. One cause of departure was attributed to the periodic wetting and unwetting of the suction side of the hydrofoil by the spray sheet.

\section{Linearized Theory of Cavity Flow}

A different approach to the cavity problem was introduced by Tulin $(1953,1955)$, who first formulated a linearized theory for the case when the body-cavity system is sufficiently thin or slender. The departure of the flow quantities from a uniform primary flow is assumed to be small so that the flow variables may be expanded about the basic flow to formulate a firstorder linear theory. The required flow quantities can then be determined completely by quadratures. This theory has been further developed by others for different problems in numerous research activities. The scope of these studies has become so wide that a brief unified discussion, as given here, would fall short of giving a complete picture; for further details see the more extensive reviews by Cooper (1958), Parkin (1959), and Tulin (1964).

To give a simple presentation of the linearized theory, we consider the incompressible potential flow past a two-dimensional, thin body-cavity system at given cavitation number $\sigma=\left(p_{\infty}-p_{c}\right) / \frac{1}{2} \rho U^{2}$. Writing for the velocity $\mathbf{q}(x, y)=U(1+u, v)$, we assume that $u^{2}+v^{2} \ll 1$ except near some isolated 
singularities. The conditions on the boundary of a finite body-cavity system, by linearization, become

$$
\begin{aligned}
& v^{ \pm}(x) \equiv v(x, 0 \pm)=s^{ \pm}(x) \quad\left(0<x<c^{ \pm}\right) \\
& u^{ \pm}(x) \equiv u(x, 0 \pm)=\frac{1}{2} \sigma \quad\left(c^{ \pm}<x<\ell\right)
\end{aligned}
$$

where $s^{+}(x), s^{-}(x)$ are the slope functions (relative to the $x$ direction) of the upper and lower wetted body surface, respectively, $c^{+}, c^{-}$are the corresponding detachment points, and $\ell$ is the undetermined cavity closure point. According to Tulin's model, the cavity closes with a singularity (represcnting a point force acting in the free-stream direction) at $z=\ell$, near which $w=u-i v$ behaves like $N(z-\ell)^{1 / 2}, N$ being a real constant. Under this closure condition, the circulation around the body-cavity system can no longer be arbitrarily prescribed. Other possible closure configurations (fully or partially open cavities) were discussed by Fabula (1962). We further note that as the location of cavity boundary is approximated by the slit $c^{ \pm}\langle x<\ell, y=0 \pm$, the the kinematic condition [Equation (4)] is relaxed in the first-order theory except for calculating the cavity shape a posterioi. The flow at infinity is regular and satisfies three conditions, namely, $w=u-i v \rightarrow 0$ and is not sourcelike as $|z| \rightarrow \infty$. In terms of the expansion

$$
\begin{aligned}
w(z)=a_{0}+i b_{0}+\left(a_{1}+i b_{1}\right) z^{-1}+\left(a_{2}+i b_{2}\right) z^{-2} & +\cdots \\
& (|z| \gg \ell)
\end{aligned}
$$

$a_{n}$ and $b_{n}$ being real coefficients, the three conditions imply that

$$
a_{0}=0, \quad b_{0}=0, \quad a_{1}=0
$$

The last one is of ten called the "closure condition." Finally, we require that $w(z)$ be continuous at a fixed detachment, and both $w$ and $d w / d z$ be continuous at a free detachment, the latter being the linearized version of the VillatBrillouin condition.

The solution of this boundary-value problem can be uniquely determined once its admissible singularities are made definite. In nonlifting flows $\left(s^{-}(x)=-s^{+}(x), c^{+}=c^{-}\right), w$ has a logarithmic singularity $w \sim\left(s^{+}(0) / \pi\right)$ $\log z$, near a pointed vertex at $z=0$, or $w$ behaves like $A z^{\prime-1}(0<\nu<1)$ near a blunt nose. For a lifting plate with a sharp leading edge, a case first treated by Tulin and later by others, the stagnation point, upon linearization, is at the leading edge (note that by the exact theory, it is at a distance of $O\left(\alpha^{4}\right)$ behind the leading edge for small incidence, $\alpha$ ); thus $c^{+}=0$, and hence by the local conformal behavior, $w \sim$ const $z^{-1 / 4}$ near the leading edge at $z=0$. This case was extended by Newman (1956) to a rounded leading edge of large curvature, near which we then have $w \sim$ const $z^{-1 / 2}$ as in thin-airfoil theory.

This problem is reduced to the Riemann-Hilbert form if the flow field in the $z$ plane is mapped conformally onto the lower half $\zeta$ plane, with the 
wetted surface mapped onto $-a<\operatorname{Re} \zeta<1, \operatorname{Im} \zeta=0$, and the cavity boundary onto the rest of the $\operatorname{Re} \zeta$ axis, by the transformation

$$
\zeta=\left(\frac{z}{c^{-}} \frac{c^{-}-\ell}{z-\ell}\right)^{1 / 2} \quad \text { with } \quad a=\left(\frac{c^{+}}{c^{-}} \frac{\ell-c^{-}}{\ell-c^{+}}\right)^{1 / 2}
$$

The solution of the resulting Riemann-Hilbert problem is

$$
\begin{aligned}
w= & \frac{\sigma}{2}+\frac{1}{\pi i}[(\zeta+a)(\zeta-1)]^{1 / 2} \\
& \cdot\left\{\int_{-a}^{1} \frac{s(x(\xi)) d \xi}{[(\xi+a)(1-\xi)]^{1 / 2}(\xi-\zeta)}+\frac{A}{\zeta}+B\right\}
\end{aligned}
$$

where $s(x(\xi))=s^{+}(x)$ for $-a<\xi<0$, and $s(x(\xi))=s^{-}(x)$ for $0<\xi<1$; the terms with real coefficients $A, B$ signify the complementary solution, admitted to provide the appropriate singularities at the leading edge $(\zeta=0)$ and at the cavity closure $(\zeta=\infty)$. For fixed detachments at given $c^{ \pm}$, the above solution involves three unknown constants, $\ell, A$, and $B$, which are to be determined by the three conditions in Equation (48). If $c^{+}$and/or $c^{-}$are unknown free detachments, they are determined by the additional condition that $d w / d z$ be continuous at each of these points. The algebraic equations for determining all these parameters, however, can be highly transcendental for general body shapes.

The lift coefficient $C_{L}$, moment coefficient (about the leading edge, positive for nose down) $C_{M}$, and cavity volume (of unit breadth) $S$, are directly related to the coefficients in Equation (47). In fact, with the chord $c$ taken to be of unit length, we have $(\mathrm{Wu}, 1957 \mathrm{a})$

$$
C_{L}=4 \pi b_{1}, \quad C_{M}=4 \pi b_{2}, \quad S=-2 \pi a_{2}
$$

For cavitating hydrofoils with a sharp leading edge, the drag coefficient is given by

$$
C_{D}=2 \pi N^{2}, \quad N=\lim _{|z-\ell| \rightarrow 0}(z-\ell)^{1 / 2} w(z)
$$

In the case when a suitably rounded leading edge becomes fully wetted so that a leading edge suction is manifest, this suction force is to be subtracted from the above formula to yield the resultant drag.

In general, the linearized theory is found to overestimate the pressure on the body surface (hence also the lift, drag, and moment) as compared with the exact theory, the discrepancy increasing with increasing cavitation number, body thickness, and angle of attack. Agreement between the approximate and exact theories is considerably improved by including in the former the second-order corrections, as shown by Chen (1962). The linear theory has been applied to different problems of interest, notably by Acosta (1955) and 
Timman (1958) to partial cavity flows, by Parkin (1957b) and Geurst (1960) to unsteady cavity flows, and by Acosta (1960) and Cohen \& Sutherland (1958) to cavitating cascades.

\section{Conclusion}

The literature on jets, cavity, and wake flows is very extensive; and much has been omitted here. One noticeable omission is a discussion of the viscous effect, although some related observational knowledge has been described in Section 2. Insofar as the far-wake is concerned, the phenomenon is relatively well understood. The laminar flow at great distances behind an obstacle has certain characteristics that can be investigated independently of the specific shape of the body. At such large distances the action of the body on the fluid may be represented by a point force (drag and lift), since the detailed distribution of the surface force on the body ceases to be of any importance. Another useful simplification is that the viscous flow far away can be adequately described by the Oseen equations as the first approximation (uniformly valid in this region) of the Navier-Stokes equations. The simple and elegant analysis of the laminar far-wake by Prandtl (1935) is now a classic. A complete evaluation of the flow field within and outside the laminar wake is available (Lagerstrom 1964). Prior to the degeneration of a turbulent farwake, its lateral dimension and mean velocity distribution can be estimated by simple dimensional arguments, according to Prandtl (1926).

The major problem of fundamental importance to wake flows is undoubtedly the viscous near-wake and the immediately ensuing reattachment process. A general theory, with proper consideration of the free shear layer, and perhaps a vortical region, is extremely desirable in order to explain and predict observational knowledge on details of flow in these regions. Further study, such as by extension of the inverse method of Catherall \& Mangler (1966), may cast more light on the mechanism. Presumably, a successful theory would provide a relationship between the free-stream Reynolds number and the wake number (or cavitation number), thus curtailing the necessary measurement of the latter. [For laminar near-wakes at moderately large Reynolds numbers, see Keller \& Takami (1966) and Roshko (1967).] Such an achievement would fulfill an outstanding final goal of the wake theory.

Investigations along this line would also depend on an accurate determination of the viscous separation. The study of the laminar separation, including the configurations of streamlines, vortex lines, and isobars, for two- and three-dimensional single-phase flows, as discussed by Lighthill (1963), is of great value. For two-phase cavity flows, similar studies, both theoretical and experimental, are urgently needed. Further extension to the turbulent separation is of both academic and practical interest.

In addition to these basic problems, special studies would also be of great significance on (i) general methods of solution to unsteady cavity and wake flows, (ii) calculation of three-dimensional free-surface flows, (iii) improved approximate theories for effective applications, (iv) optimum-shape prob- 
lems in free-surface flows, and (v) hydrodynamic stability of viscous flows having free boundaries. Undoubtedly, other problems of fundamental value may occur to readers of this survey; all combined they would serve to elaborate the foresighted observation of Lord Rayleigh.

\section{ACKNOWLEDGMENTS}

I take pleasure in expressing my appreciation and gratitude to Dr. Christopher Brennen for valuable and extensive discussions on many topics covered in this survey. Sincere thanks are due from me to the Office of Naval Research, Fluid Dynamics Branch, for its continued support, including that given to the preparation of this article. 


\section{LITERATURE CITED}

Ablow, C., Hayes, W. 1951. Perturbation of free surface flows. Tech. Rep. No. 1, Grad. Div. Appl. Math., Brown Univ.

Acosta, A. J. 1955. A note on partial cavitation of flat plate hydrofoils, Calif. Inst. Technol. Hydrodyn. Lab. Rep. E-19.9

Acosta, A. J. 1958. The effect of a longitudinal gravity field on the supercavitating flow over a wedge. Calif. Inst. Technol. Eng. Div. Rep. 70.1

Acosta, A. J. 1960. Cavitating flow past a cascade of circular arc hydrofoils. Calif. Inst. Technol. Rep. E-79.2

Apelt, C. J. 1957. Some siudies of fluid flow at low Reynolds numbers. Thesis, Oxford Univ.

Arakeri, V. 1971. Water tunnel investigations of scale effects in cavitation detachment from smooth slender bodies and characteristics of flow past a bi-convex hydrofoil. Calif. Inst. Technol. Rep. E-79A.12

Arie, M., Rouse, H, 1956. J. Fluid Mech. $1: 129-41$

Armstrong, A. H. (appendix by Clark, C. G.) 1954. Drag coefficients of wedges and cones in cavity flow. Armament Res. Estab. Rep. 21/54

Armstrong, A. H., Dunham, J. H. 1953. Axisymmetric cavity flow. Armament Res. Estab. Rep. $12 / 53$

Batchelor, G. K. 1956. J. Fluid Mech. 1:388-98

Benjamin, T. B. 1964. J. Fluid Mech. 19; 137-44

Bergman, S. 1955. J. Rat. Mech. Anal. 4: 883-905

Birkhoff, G. 1950. Hydrodynamics. Princeton, N.J.: Princeton Univ. Press

Birkhoff, G., Zarantonello, E. H. 1957. Jets, Wakes and Cavities. New York: Academic

Brennen, C. 1969a. J. Fluid Mech. 37:11527 (1 plate)

Ibid. $1969 \mathrm{~b}$. $671-88$

Ibid. 1970a. 44:33-49 (2 plates)

Ibid. 1970b. 51-63 (2 plates)

Brillouin, M. 1911. Ann. Chim. Phys. 23: 145-230

Brodetsky, S. 1923. Proc. Roy. Soc. London Ser. A 102:542-53

Calvert, J. R. 1967a. J. Fluid Mech. 27:27389

Ibid. 1967b. 29:691-703 (1 plate)

Carmody, T. 1964. Trans. ASME J. Basic Eng. 86:869-82

Catherall, D., Mangler, K. W. 1966. J. Fluid Mech. 26:163-82

Chaplygin, S. 1904. Ann. Sci. Imp. Univ., Math.-Phys. Cl. $1904: 1-121$. Also 1948.
Sobranie Sochinenii, Tom II, 19-137. Moscow: Gos. Izdat. Tekh.-Teor. Lit. Transl. 1944. NACA TN 1063

Chen, C. F. 1962. J. Fluid Mech. 13:321-32

Chuang, F.-K. 1955. Acta Sci. Sinica 4, no. $2: 277-96$

Coburn, N. 1945. Quart. Appl. Math. 3:10616

Cohen, H., Sutherland, C. D. 1958. Finite cavity cascade flow. Rensselaer Polytech. Inst. Math, Rep. 14

Cooper, R. D., editor. 1958. Proc. Second Symp. Naval Hydrodyn. Washington, DC

Cox, A. D., Clayden, W. A., 1958. J. Fluid Mech. 3:615-37

Cumberbatch, E. 1961. J. Ship Res. 4, no. 4:1-8

Cumberbatch, E., Wu, T. Y. 1961. J. Fluid Mech. 11:187-208

Curle, N. 1956. Proc. Roy. Soc. London Ser. A 235:375-81; 382-95

Dagan, G. 1971. J. Fluid Mech. 49:179-92

Delong, R. K., Acosta, A. J. 1969. Experimental investigation of nonsteady forces on hydrofoils oscillating in heave. Calif. Inst. Technol, Rep.

Efros, D. A. 1946. Dokl. Akad. Nauk SSSR, $51: 267-70$

Eisenberg, P., Pond, H. C. 1948. Water tunnel investigations of steady state cavities. David Taylor Model Basin Rep. 668

Eisenberg, P., Tulin, M. P. 1961. In Handbook of Fluid Dynamics, ed. V. L. Streeter, Sec. 12, New York: McGraw-Hill

Eppler, R. 1954. J. Rat. Mech. Anal. 3:591644

Fabula, A. G. 1962. J. Fluid Mech. 12:22740

Fage, A., Johansen, F. C. 1927. Proc. Roy. Soc. London Ser. A 116:170-97

Fisher, D. D. 1963. J. Math. and Phys. 42: 14-26

Fisher, J. W. 1944. The drag on a circular plate generating a cavity in water. Underwater Ballistics Res. Comm. Rep. No. 17

Fox, J., Morgan, G. 1954. Quart. Appl. Math. 11:439-56

Frankl, F. I. 1945. Izv. Akad. Nauk SSSR, Ser. Mat. 9:121-43; 387-422

Furuya, O., Acosta, A. J. 1972. A Note on the Calculation of $H y d r o f o i l s$ with Rounded Noses. Japan Soc. Mech. Eng. Second Int. Turbomachinery Conf., Tokyo, Japan, September 4-11, 1972

Gadd, G. E. 1962. Two-dimensional separated or cavitating flow past a flat plate normal to the stream. Aeronaut. Res. Counc. Curr, Pap. No. 697

Garabedian, P. R. 1956. Pac. J. Math. 
6:611-84; Bull. Amer. Math. Soc. 62:21935

Gaster, M. 1971. J. Fluid Mech. 46:749-56

Gerber, R. 1951. C.R. Acad. Sci. Paris 233: 1560-62

Gerrard, J. H. 1966. J. Fluid Mech. 25 :40113

Geurst, J. A. 1960. Arch. Rat. Mech. Anal. $5: 315-46$

Gilbarg, D. 1949. Proc. Nat. Acad. Sci. USA 35:609-12

Gilbarg, D. 1952. Z. Angew. Math. Phys. 3:34-42

Gilbarg, D. 1960. In Handbuch der Physik, Vol. 9, 311-445, Berlin: Springer-Verlag.

Goldburg, A., Washburn, W. K., Florsheim, B. H. 1965. AIAA J. 3:1332-33

Goldstein, S. 1969. Ann. Rev. Fluid Mech. $1: 1-28$

Görtler, H. 1942. Z. Angew. Math. Mech. $22 ; 244-54$

Grange, J. M., Kineberg, J. M., Lees, L. 1967. AIAA J. 5:1089-96

Gurevich, M. I. 1961. Theory of Jets in Ideal Fluids (in Russian). Moscow: Gos. Izdat. Fiz.-Mat. Lit. English trans. 1965. New York: Academic

Helliwell, J., Mackie, A. G. 1957. J. Fluid Mech. 3:93-109

Helmholtz, H. 1868. Monatsber. Akad. Wiss. Berlin, 23:215-28

Hopkinson, B. 1898. Proc. London Math. Soc. 29:142-64

Hsu, E-Y., Perry, B. 1954. Water tunnel experiments on spheres in cavity flow. Calif. Inst. Technol. Rep. No. E-24.9

Imai, I. 1957. Theory of Bluff Bodies. Univ. Maryland. Inst. Fluid Dyn. Appl. Math. See also 1960. Proc. 10th Int. Congr. Appl. Mech., Stresa, 123-24

Jacob, C. 1946. A cad. Roum. Bull. Sect. Sci. 28:637-41

Joukowsky, N. E. 1890. Mat. Sbornik (Rec. Math.) 15:121-278. Also 1949. Sobranie Sochinenii, Tom II, 489-626. Moscow: Gos. Izdat. Tekh.-Teor. Lit.

Kaplun, S. 1967. Fluid Mechanics and Singular Perturbations. New York: Academic

Ke.ller, H. B., Takami, H. 1966. In $\mathrm{Nu}$ merical Solutions of Nonlinear Differential Equations, ed. D. Greenspan, 115-140. New York: Wiley

Kelvin, Lord (Wm. Thompson). 1880. Phil. Mag. 10:155-68

Kirchhoff, G. 1869. J. Reine Angew. Math. $70: 289-98$

Klose, G. J., Acosta, A. J. 1969. J. Ship Res. 13:92-102

Knapp, R. T., Daily, J. W., Hammitt, F. G. 1970. Cavitation. New York: McGrawHill
Kovasznay, L. S. G. 1949. Proc. Roy, Soc, London Ser. A 198:174-90

Kreisel, G. 1946. Cavitation with finite cavitation numbers. Admirally Res. Lab. Rep. $R 1 / H / 36$

Kuznetsov, A. V., Kiselev, O. M., Kotlyar, L. M. 1971. Theoretical study of nonlinear problems of liquid flow with free boundaries. Proc. IUTAM Symp. on Non-steady Flow of Water at High Speeds, Leningrad, USSR

Lagerstrom, P. A. 1964. In High Speed Aerodynamics and Jet Propulsion, ed. F. K. Moore, Vol. 6, Sec. B. Princeton, N. J.: Princeton Univ. Press

Larock, B. E., Street, R. L. 1967. J. Fluid Mech. 29:317-36

Lavrentiev, M. A. 1962. The Variational Method in Boundary Problems for Systems of Equations of Elliptic Type. Moscow: Akad. Nauk SSSR

Lees, L., Kubota, T. 1957. J. A ero. Sci. 24: 195-202

Lees, L., Reeves, B. L. 1964. AIAA J. 2: 1907-20

Leray, J. 1935. Comment. Math. Helv. 8:149$80 ; 250-63$

Leray, J., Schauder, J. 1934. Ann. Sci. Ecole Norm. Sup. 51:45-78

Levi-Civita, T. 1907. Rend. Circ. Mat. Palermo 18:1-37.

Levinson, N. 1946. Ann. of Math. 47:70430

Lewy, H. 1952. Comm. Pure Appl. Math. $5: 413-14$

Liepmann, H. W., Laufer, J. 1947. NACA TN 1257

Lighthill, M. J. 1945. A note on cusped cavities. Aeronaut. Res. Counc. Rep. and Memo. 2328

Lighthill, M. J. 1963. In Laminar Boundary Layers, ed. L. Rosenhead, Chap. II. Oxford: Clarendon Press

Mackie, A. G. 1958. Proc. Cambridge Phil. Soc. 54:538-53

McNown, J. S., Yih, C.-S., editors. 1953. Free-streamline analysis of transition flow and jet deflection. State Univ. of Iova, Iowa City, Eng. Bull. 35

Meijer, M. C. 1959. Int. Shipbuilding Progr. 6:361-68

Meijer, M. C. 1967. J. Ship Res. 11:170-89

Nekrasoff, N. A. 1922. Izv. IvanovoVoznesensk. Politekh. Inst. No. 5:3-19. Also Sobranie Sochinenii, Tom I, 52-68. Moscow: Akad. Nauk SSSR

Newman, J. N. 1956, Supercavitating flow past bodies with finite leading edge thickness. David Taylor Model Basin Rep. 1081

Oudart, A. 1949. Publ. Sci. Tech. Min. Air No. 234 
Pai, S. I. 1954. Fluid Dynamics of Jets. New York: Van Nostrand

Parkin, B. R. 1957a. A note on the cavity flow past a hydrofoil in a liquid with gravity. Calif. Inst. Technol. Eng. Div. Rep. No. 47-9

Parkin, B. R. 1957b. Fully cavitating hydrotoils in nonsteady motion. Calif. Inst. Technol. Eng. Div. Rep. No. 85-2

Parkin, B. R. 1958. J. Ship Res. 1, no. 4: 34-56

Parkin, B. R. 1959. Linearized theory of cavity flow in two dimensions. The RAND Corp. Rep. No. P-1745, Santa Monica, Calif.

Plesset, M. S. 1964. In Cavitation in Real Liquids, ed. Robert Davies, 1-18, Amsterdam: Elsevier

Plesset, M. S., Shafier, P. A., Jr. 1948. J. Appl. Phys. 19:934-39

Prandt, L. 1904a. Verhandl. d. 3ten Int. Malh.-Kangr. Heidelberg, 484-91. Leipzig: Teubner

Prandtl, L. 1904b. Phys, Z. 5:599-601

Ibid. 1907. 8:23-32

Prandtl, L. 1926. Proc. 2nd Int, Congr. Appl. Mech. Zurich, 62-74

PrandtI, L. 1935. In Aerodynamic Theory, ed. W. F. Durand, Vol. III, 34-208. Berlin: Springer-Verlag

Pykhteev, G. N. 1960. Prikl. Mat. Mekh. 24:157-61

Rayleigh, Lord. 1876. Phil. Mag. (5) 2:43014:56-63, disc. 63-67. Also 1900. Papers on Mechanical and Physical Subjects, vol. 1, 51-58

Reeves, B. L., Lees, L. 1965. AIAA J. 3: 2061-74

Reichardt, H. 1942. Gesetzmässigkeiten der freien Turbulenz. VDI-Forschungsheft No. 414

Reynolds, O. 1873. Trans. Inst. Naval Arch. 14:56-63, disc. 63-67. Also 1900. Papers on Mechanical and Physical Subjects, Vol. $1,51-58$

Reynolds, O. 1883. Phil. Trans. Roy. Soc. London 174:935-82. Also 1900. Papers, vol. 2, 51-105

Riabouchinsky, D. 1920. Proc. London Math. Soc. 19:206-15

Richardson, A. 1920. Phil. Mag. (6) 40:97-110

Rispin, P. P. 1967. A singular perturbation method for nonlinear water waves past an obstacle, PhD thesis. Calif. Inst. Technol., Pasadena, Calif.

Robertson, J. M., Wislicenus, G. F., editors. 1969. Cavitation Slate of Knonledge. New York: Am. Soc. Mech. Eng.

Roshko, A. 1954a, NACA TN 3168

Ibid., 1954b. NACA TN 3169

Roshko, A. 1955, J. Aero. Sci. 22:124-32

Roshko, A. 1961. J. Fluid Mech. 10:345-56
Roshko, A. 1967. Proc. Canadian Congr. Appl. Mech. Quebec vol. 3, 3-081-115

Roshko, A., Fiszdon, W. 1967. In Problems of Hydrodynamics and Continuum Mechanics, 606-16. Philadelphia: Soc. Ind. Appl. Math.

Sakurai, T. 1956. J. Phys. Soc. Japan 11: 710-15

Salamotov, D. 1959. Prikl. Mat. Mekh. 23: 361-9. Transl. J. Appl. Math. Mech. 23: 508-19

Sedov, L. I. 1949. Vestn. Moskov. Univ. 4, no. 9:3-14

Sedov, L. I. 1966. Plane Problems in Hydrodynamics and Aerodynamics, 2nd. ed. (in Russian). Moscow: Izdat. "Nauka"

Shen, Y. T., Ogilvie, T. F. 1972. J. Ship Res. 16:3-21

Shiffman, M., Spencer, D. 1951. Comm. Pure Appl. Math. 4:379-417

Silberman, E. 1959. J. Fluid Mech. 5:33754 (2 plates)

Skripkin, N. A. 1955. Prikl. Mat. Mekh. $19: 89-98$

Southwell, R. V., Vaisey, G. 1946. Phil. Trans. Roy. Soc. London. Ser. A 240:11761 (2 plates)

Struck, H. G. 1970. NASA TN D-5634

Thom, A. 1933. Proc. Roy. Soc. London Ser. A 141:651-69

Thoma, D. 1926. Hydraulische Probleme, 65-74. Berlin: VDI Verlag

Timman, R. 1958, A general linearized theory for cavitating hydrofoils in nonsleady flow. Prac. Second Symp. Naval Hydrodyn., Washington, DC, 559-82

Trefftz, E. 1916. Z. Math. Phys. 64:34-61

Tsien, H. S. 1939. J. Aero. Sci. 6:399-407

Tulin, M. P. 1953. Steady two-dimensional cavity flows about slender bodies, David Taylor Model Basin Rep. 834

Tulin, M. P. 1958. Discussion of the paper by $R$. Timman (1958), loc. cit., p. 580

Tulin, M. P. 1964. J. Ship Res. 7, no. 3: 16-37

Tulin, M. P., Burkart, M. P. 1955. Linearized theory for flows about lifting foils at zero cavitation number, David Taylor Model Basin Rep. C-638

Villat, H. 1914. J. de Math. (6) 10:231-90

von Karman, T. 1949. Ann. Mat. Pura Appl. 29:247-49; Also 1956. Collected Works, Vol. 4, 396-98. London:Butterworths

Wang, D. P. 1970. An unsteady cavity flow. Proc. Eighth Symp. Naval Hydrodyn., Pasadena, Calif., 189-216

Wang, D. P., Wu, T. Y. 1963. Arch. Rat. Mech, Anal. 14:127-52

Wang, D. P., Wu, T. Y. 1965. Trans. ASME, J. Basic Eng. 87:1006-10

Wehausen, J. V. 1965. In Research Frontiers 
in Fluid Dynamics, ed. R. J. Seeger, G. Temple, Chap. 18. New York: Interscience

Weinig, F. 1956. Proc. Ninth Int. Congr. Appl. Mech., Vol. 1, 215-20, Brussels: Univ. of Brussels

Woods, L. C. 1951. Quart. J. Mech. Appl. Math. 4:358-70

Woods, L. C. 1954. Aeronaut. Res. Counc. Curr. Pap. No. 149

Woods, L. C. 1955. Proc. Roy. Soc. London Ser. A 229:152-80

Ibid. 1957. 239:328-37

Woods, L. C. 1961. The Theory of Subsonic Plane Flow. Cambridge: Cambridge Univ. Press

Woods, L. C. 1964. J. Fluid Mech. 19: 124-36.

Wu, T. Y. 1956. J. Math and Phys. 35: 236-65

Wu, T. Y. 1957a. A simple method for calculating the drag in the linear theory of cavity flows, Calif. Inst. Technol. Eng. Div. Rep. 85-5
$\mathrm{Wu}, \mathrm{T}, \mathrm{Y}, 1957 \mathrm{~b}$. A linearized theory for nonsteady cavity flows, Calif. Inst. Technol. Eng. Div. Rep. 85-6

Wu, T. Y. 1958. Unsteady cavitating flowes. Proc. Second Symp. Naval Hydrodyn., Washington, DC, 293-315

Wu, T. Y. 1962. J. Fluid Mech. 13:161-81

Wu, T. Y. 1967. Int. Shipbuilding Progr. $14: 88-97$

Wu, T. Y. 1968. In Basic Developments in Fluid Dynamics, ed. M. Holt, 1-116. New York: Academic

Wu, T. Y., Wang, D. P. 1964a. J. Fluid Mech. 18:65-93

Wu, T. Y., Wang, D. P. 1964b. Trans, ASME, J. Basic Eng. 86:556-60

Wu, T. Y., Whitney, A. K., Lin, J. D. 1969. Wall effect in cavity flows. Calif. Inst. Technol. Rep. No. E-111A.5

Wu, T. Y., Whitney, A. K., Brennen, C. 1971. J. Fluid Mech. 49:223-56

Yih, C-S. 1960. Proc. Roy. Soc. London Ser. A 258:90-100

Young, J. O., Holl, J. W. 1966. Trans. ASME, J. Basic Eng. 88:163-76 


\section{CONTENTS}

As Luck Would Have It-A Few Mathematical Reflections, Henri Villat.

Fluid Mechanics of Heat Disposal from Power Generation, Donald R. F. Harleman and Keith D. Stolzenbach . . . . . 7

Mantle Convection and the New Global Tectonics, Donald $L$. Turcotte and E. R. Oxburgh . . . . . . . . . . . . 33

Finite Amplitude Disturbances in the Flow of Inviscid Rotating and Stratified Fluids over Obstacles, Robert R. Long . . 69

Locomotion of ProtozoA, Theodore L. Jahn and John J. Votta . . 93

'Magnetohydrodynamics of the Earth's Core, $P$. H. Roberts and A. M. Soward . . . . . . . . . . . . . . . 117

Chemically Reacting Flows, Ernst Becker. . . . . . . . 155

Vortex Breakdown, M. G. Hall . . . . . . . . . . . 195

Self-Gravitating Gaseous Disks, C. Hunter . . . . . . . 219

Cavity and Wake Flows, Th. Yao-tsu $W u$. . . . . . . . . 243

Self-Similar Solutions as Intermediate Asymptotics, G. I. Barenblatt and Ya. B. Zel'dovich. . . . . . . . . . . . . 285

Periodic Flow Phenomena, Eberhard Berger and Rudolf Wille . . 313

Oil Spreading on the Sea, David P. Hoult. . . . . . . . 341

One-Dimensional Flow of Liquids Containing Small Gas BubBLES, L. van Wijngaarden. . . . . . . . . . . . . . . 369

Sailing Vessels and Sails, Jerome H. Milgram . . . . . . 397

Wing-Body Aerodynamic Interaction, Holt Ashley and William $P$. Rodden . . . . . . . . . . . . . . . . . 431

Bounds on Flow QuantTties, Louis N. Howard . . . . . . . 473

INDEXES

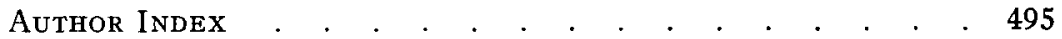

Cumulative Index of Contributing Authors, Volumes 1-4 . 502

Cumulative Index of Chapter Titles, Volumes 1-4 . . . 503 